\title{
Zehn Sekunden, die über die Zukunft der Hochschulen entscheiden
}

Sekunden sind in der Musik nebeneinanderliegende Ganztöne. Erklingen sie zusammen, hört man einen dissonanten Klang - etwas reibt sich dabei ganz offensichtlich, will sich auflösen, strebt in einen anderen Zustand. Dissonanzen in der Musik haben eine dynamische Kraft, sie erscheinen als instabiler Zustand, sind kein Ruhepol. Kein Moment des Verweilens - sie wollen weiter. Sie scheinen einen nächsten Schritt notwendig zu machen, und zeigen in der Musik in eine Richtung. Und doch sind sie die kleinste Einheit großer Musikstücke, aller Musikstücke. Die Hochschule der Zukunft steht vor der Frage, ob sie die sich derzeit abzeichnenden Dissonanzen als Entwicklungsmomente verstehen kann, aus denen heraus sie eine neue Architektur komponieren, sie als Potenziale für Entwicklung verstehen kann.

Was sind diese Sekunden? Diejenigen Entwicklungen, die einerseits Probleme, Schwierigkeiten, Herausforderungen darstellen, zu Dissonanz führen und andererseits gleichzeitig damit Entwicklungen herausfordern? Welches sind die zehn Sekunden, die über die Zukunft der Hochschule entscheiden?

Die Zukunft der Hochschule spannt sich wie ein Horizont. Luhmann (1976) beschreibt, dass in allen sozialen Systeme Erwartungen gebildet werden, die maßgeblich sind dafür, wie sich das System, auch die Hochschule, in seinen Operationen auf die Zukunft ausrichtet. Daher ist es wichtig, für die Zukunft der Hochschule auch die Situation innerhalb der Hochschule und die Erwartungen ihrer Akteurinnen und Akteure mit einzubeziehen. Niklas Luhmann (ebenda) unterscheidet in diesem Zusammenhang zwei Aspekte, nämlich gegenwärtige Zukünfte - also Projektionen, etwa in Gestalt von Utopien - und zukünftige Gegenwarten in Gestalt von technologischen Orientierungen, kausalen oder stochastischen Verbindungen zukünftiger Ereignisse. Die vorliegende Arbeit versteht sich als Beitrag zur zukünftigen Gegenwart der Hochschulen. 
Es ist eine Vielzahl von unterschiedlichen Entwicklungen, gesellschaftlicher, wirtschaftlicher, politischer und technologischer Art, die dazu führen, dass sowohl ein Transformationsklima als auch eine Transformationsnotwendigkeit entsteht. Dabei stechen einige Aspekte wie weit sichtbare Landmarken heraus und bilden Anlässe für kleinere und größere Krisen und damit neue Entwicklungen. Zehn Punkte werden im Folgenden herausgegriffen und analysiert, mit dem Ziel, herauszuarbeiten, in wieweit sie Transformationsdruck auf Hochschulen ausüben.

\section{C1.1 Erste Sekunde: Digitalisierung - Hochschulbildung in einer digitalen Welt}

Die Digitalisierung ist - auch für Hochschulen - eine derart mächtige Entwicklung, dass es sich sicher lohnen würde, dem Einfluss der Digitalisierung auf die Hochschulbildung ein eigenes Buch zu widmen. Vielfältige Publikationen zeugen davon. Jedoch zeigt sich in der aktuellen Diskussion um Hochschulstrategien, dass die Digitale Transformation nicht ein Ziel an und für sich ist. Es zeigt sich, dass immer weniger Hochschulen sich eine digitale Strategie geben, und immer mehr dazu übergehen, Digitalisierung als Mittel zu verstehen, um das eigene Hochschulprofil strategisch zu überdenken oder zu schärfen. Schünemann und Budde (2018) arbeiten pointiert heraus, dass das das Ergebnis dabei oftmals in einer Strategie für Hochschulbildung in einer digitalen Welt besteht, aber eben gerade nicht in einer Strategie für Digitalisierung.

Digitale Bildung ist gleichzeitig das burning issue der derzeitigen Diskussion über die Hochschule der Zukunft. Es ist Gegenstand unzähliger Gespräche, Diskussionen, Konzeptionspapiere und wissenschaftlicher Untersuchungen. Sowohl in der Bildungspolitik als auch in der gegenwärtigen Hochschuldebatte sowie in der bildungswissenschaftlichen Debatte und vielfältigen anderen Diskussionskontexten. Dabei hat die Diskussion über digitale Bildung eine Konjunktur hinter sich, die sich auch kritisch mit den Begrifflichkeiten auseinandersetzt und in jüngster Zeit auch stärker auf den Bildungsprozess als solchen fokussiert. Immer weniger wird von digitaler Bildung gesprochen, sondern vielmehr auf Bildung in der zukünftigen Gesellschaft rekrutiert oder auf Bildung in einer digitalen Gesellschaft oder unter Bedingungen der Digitalisierung gesprochen (siehe z. B. die letzte Publikation des BMBF zu dem Thema: „Bildung in einer digitalen Gesellschaft“). In den Hochschulen stellt sich die Frage: Wie geht man mit den neuen Möglichkeiten um? Die neuen Möglichkeiten bieten sich entlang einer Reihe von unterschiedlichen Dimensionen. So führt Digitalisierung zu Entgrenzungsprozessen akademischer Bildung und ihrer Organisation, die auf alle Bereiche der Hochschule einen Einfluss hat: 
Das für ein akademisches Studium notwendige Wissen wird zunehmend frei digital verfügbar und ist auch von einer spezifischen akademischen Institution und ihren Akteurinnen und Akteuren entkoppelt zu erreichen. Die Koppelung von Wissenszugang und Institutionszugehörigkeit löst sich mehr und mehr auf. So ist beispielsweise ein Patchworkstudium mit unterschiedlichen akademischen Lehrveranstaltungen an unterschiedlichen Institutionen prinzipiell denkbar und wird auch zunehmend realisiert.

- Wissensvermittlungsprozesse verlieren ihre Raum- und Zeitgebundenheit und Studium kann neu und unabhängig von Seminarräumen und Präsenzveranstaltungen organisiert werden.

- Die Generierung neuen Wissens über Forschungsprozesse ist heute ohne digitale Medien und durch sie unterstützte Prozesse nicht mehr denkbar. Auch für die Interaktion zwischen Lehrenden und Lernenden, sowohl bei der Lehre als auch bei der Organisation des Studiums, werden zunehmend digitale Medien genutzt.

- Forschende, Lehrende und Studierende treten über digitale Medien zunehmend auch in einen globalen Austausch und Studium, Lehre und Forschung internationalisieren sich.

Die hier genannten Punkte stellen nur eine kleine Auswahl von Aspekten dar, die durch Digitalisierung in der Hochschule der Zukunft beeinflusst werden. Die Tatsache, dass mehr und mehr Universitäten Konzeptionen zur Digitalisierung in ihre Strategiebildungsprozesse aufnehmen, trägt dieser Entwicklung Rechnung und ist gleichzeitig Ausdruck davon (Hochschulforum Digitalsierung 2016).

Die steigende Individualisierung von akademischen Bildungsprozessen und die Vielfalt von Ansprüchen, Zielen und Methoden des Studierens wird durch die Unterstützung der Lehre und des Studiums mit digitalen Medien im oben beschriebenen Sinne erst möglich. Die Digitalisierung wirkt wie ein Ermöglicher für die Anforderungen, die eine gesteigerte Bildungsbeteiligung mit sich bringen.

Die Digitalisierung der Hochschulbildung als Technisierung zu verstehen, wäre verkürzt und falsch. In ihrem Kern stehen Aspekte wie der freie Zugang zu Wissen, Wissensressourcen, entgrenzten Kommunikationsmöglichkeiten und Vernetzung. Es stellt sich nunmehr verstärkt die Frage, wie Bildungsprozesse aussehen müssen, wenn sie eben nicht mehr auf dem schon eingeübten Hierarchiegefälle der Lehrenden als den Wissensträgerinnen und Wissensträgern einerseits und den Studierenden als den Wissensempfängerinnen und Wissensempfängern andererseits, ruhen kann. Vielmehr scheint das alte Ideal der Gemeinschaft der Studierenden und Lehrenden mit dem Ziel, innovative Ansätze durch Diskurs hervorzubringen, nun 
wieder aufscheinen zu können - im gemeinsamen Diskurs Problemszenarien zu entwickeln und zu bearbeiten.

\section{1.1.1 Beschleunigte Innovationszyklen - Wandel als das neue Normal}

Digitalisierung ist auch deshalb ein so starker Einflussfaktor, weil sich technologische Innovationszyklen immer stärker beschleunigen. Betrachtet man alleine die technische Entwicklung und stellt man sich vor, dass die letzten eintausend Jahre auf 24 Stunden geschrumpft seien, so würde die Entwicklung des Buchdruckes erst in Stunde 13, kurz nach mittags, passiert sein, die Fotografie vor ca. vier Stunden, Telefon und Radio vor etwa drei Stunden, das World Wide Web erst vor einer halben Stunde und Dienste wie Facebook, Twitter, WhatsApp sowie das iPhone an sich erst vor zehn Minuten (siehe Abbildung 23).

Gleichzeitig nimmt die Intensität der Auswirkung der unterschiedlichen beschriebenen Technologien immer weiter zu. Das heißt, wir stehen vor einer Entwicklung, in der Technologien sich immer schneller entwickeln und die Auswirkungen, die diese Technologien haben, immer schneller wirksam und gesellschaftlich spürbar werden. In allen gesellschaftlichen Bereichen entsteht der Eindruck einer „5 Minuten vor 12" Situation. Mit dem Zukunftsforscher Peter Kruse können wir hier von einem Paradigmenwechsel einer linearen hin zu einer nicht-linearen, emergenten Systemdynamik sprechen (Kruse 2009). Dabei wird die Fähigkeit des Erkennens und Reflektierens von Zusammenhängen wichtiger als das Definieren von Zielen und das Ausführen von Planungsvorgängen.

Der durch Digitalisierung hervorgerufene Wandel bewirkt ein Gefühl eines permanenten Wandels gesellschaftlicher Abläufe und Möglichkeiten. Während Änderungszyklen bisher zu neuen Zuständen beispielsweise in Organisationen oder gesellschaftlichen Entwicklungen führten, die dann eine Zeit lang den neuen Status Quo darstellten, so avancieren Änderung, Wandel und Transformation zunehmend zum neuen Normalzustand. Das Gefühl, des „5 Minuten vor 12“ wird nun zum gesellschaftlichen Grundgefühl, zum organisationalen Normal. Auch in Hochschulen ist die Agenda der beteiligten Akteurinnen und Akteure, Wissenschaftlerinnen und Wissenschaftlern sowie den Gremien zunehmend mehr auf Wandel und weniger auf Beständigkeit aus. Es gibt keinen eingeschwungenen Zustand mehr. Neue Änderungen ergeben sich dabei aus derzeit laufenden Änderungszuständen.

Für Hochschulen, ihre Curricula und die Entwicklung von Studienangeboten auch von Bedeutung ist die Auswirkung der Digitalisierung auf den Arbeitsmarkt: Nicht zuletzt werden die Geschwindigkeit der technologischen Entwicklung und ihre 


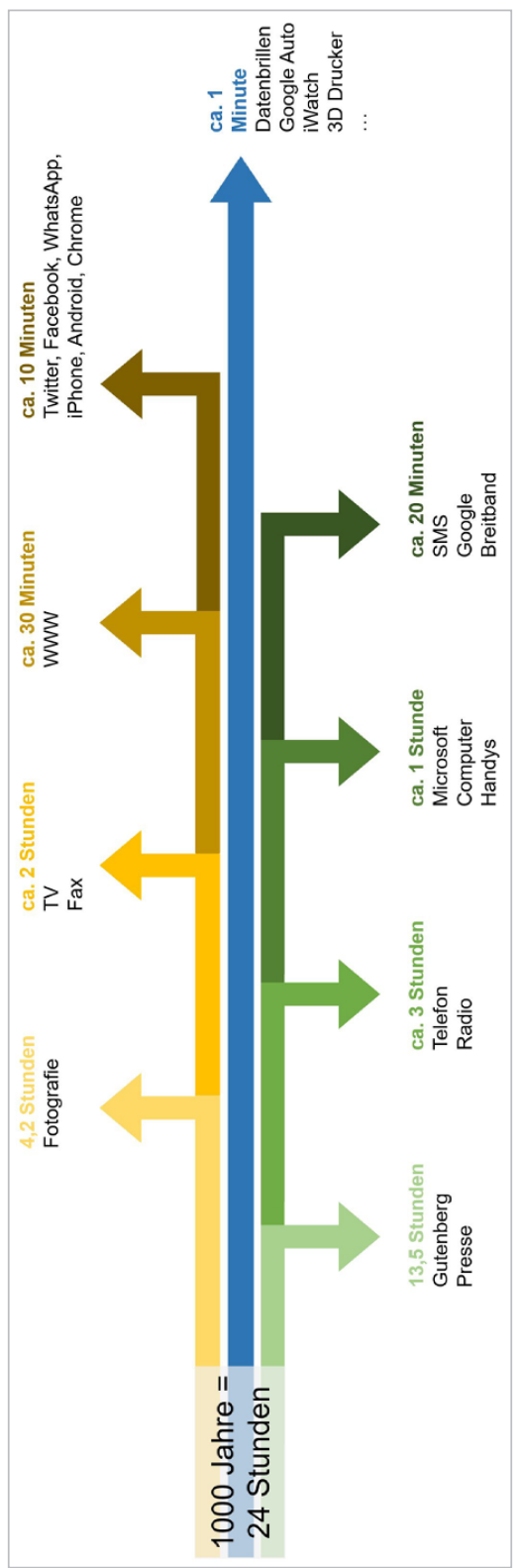

Abb. 23 Geschwindigkeit digitaler Entwicklung (inspiriert durch Ibrahim Evsan 2015) 
Auswirkungen auch durch die Diskussion deutlich, wie sie auf den Arbeitsmarkt und das Arbeitsumfeld wirkt. Die Botschaft ist häufig: Technologienentwicklungen fressen Arbeitsplätze und die Frage scheint nicht mehr zu sein, ob Arbeitsplätze wegfallen, sondern wie viel. Dabei ist im Bereich der Arbeitsmarkteffekte von Technologisierung, Robotik und künstlicher Intelligenz klar, dass überall dort wo manuelle Routinetätigkeiten ausgeübt werden, hohe Potentiale technologischer Transformation bestehen und überall dort, wo nicht routinierte soziale Fähigkeiten gefragt sind nur ein geringes Potential technologischer Substitution besteht (siehe Abbildung 24).

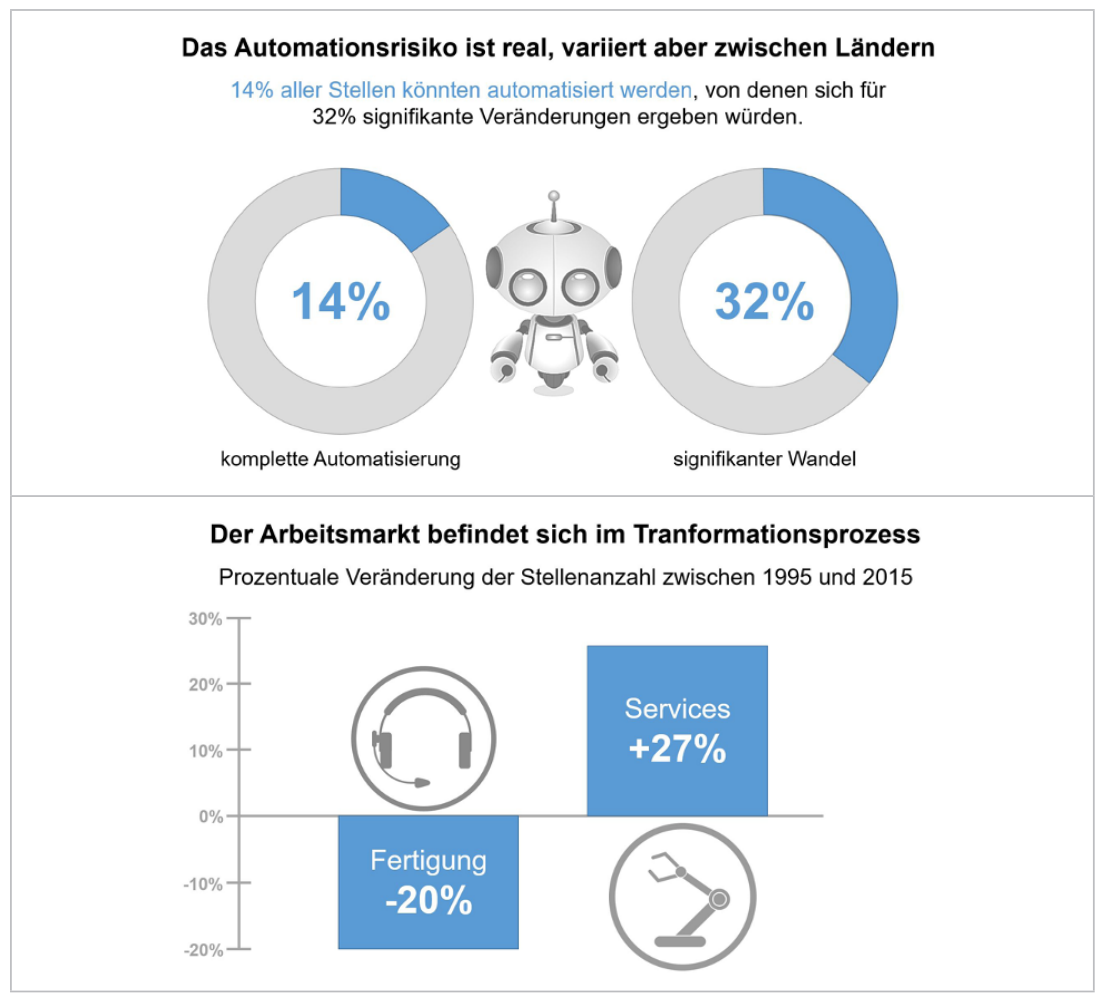

Abb. 24 Auswirkung der Digitalisierung auf den Arbeitsmarkt (Eigene Abbildung nach Daten aus OECD 2019) 
Tatsächlich ist die Frage was für ein Substitutionspotential durch Technologie besteht eine in vielen Arbeitsmarktbereichen dramatische aber derzeit noch unklare. Können auf der einen Seite zwar Tätigkeitbündel durch Computer und Maschinentechnologie substituiert werden, so stellt sich andererseits die Frage in welcher Fristigkeit und in welchem Zeitraum hierdurch sich veränderte, neue Professionsprofile bilden.

\section{C1.1.2 Umgedrehte Innovationsrichtung}

Obwohl Digitalisierung ein starkes Disruptionspotenzial mit sich bringt und in viele gesellschaftliche Teilbereiche hinein Veränderungen bewirkt, ist die Implementierung von digitalen Technologien sowie auch die Anpassung von Abläufen in Institutionen herausfordernd (Hochschulforum Digitalisierung 2016). Digitale Technologien sind zwar mittlerweile in vielen Bereichen stark integriert, jedoch ging das in vielen Fällen auch mit starken Umbrüchen einher. So ist der Handel mit Musik stark davon betroffen und hat sich in den letzten Jahren sehr dynamisch weiterentwickelt und quasi revolutioniert. Auch der Buchhandel wurde durch das Internet stark verändert. Einzelne Titel und einzelne Musikstücke können bezogen werden; eine Möglichkeit, die vorher undenkbar war. Jedoch ist es bemerkenswert, dass der Anstoß zu Neuerungen im Vertrieb oder in der Produktion immer von außen kam, nie von den Branchen selber entwickelt wurde - weder im Buchhandel noch in der Musikindustrie noch in anderen Branchen. Die Impulse zur Veränderung kamen tatsächlich immer von außen durch technologische Entwicklungen: Es waren nicht die Buchhändler die sich zusammengesetzt haben, um darüber nachzudenken, wie sie möglicherweise eine neue Form des Buchvertriebs für granulare Angebote auf Kapitel- oder Seitenebene entwickeln könnten und möglichweise sogar frei verfügbar an ganz neue Kundengruppen abgeben könnten. Sondern es war das Internet mit seinen Möglichkeiten, die Technologie die verfügbar war, die zu diesen Fragen geführt hat: Innovationen durch externe Impulse.

Betrachten wir die Hochschulen, können wir die Frage stellen, welche Auswirkungen die Digitalisierung wohl für diese Institution am Ende haben wird. Dabei ist in Hochschulen ein ähnlicher Prozess zu beobachten. Öffentlich finanzierte Hochschulen haben nur einen geringen Marktdruck. Trotzdem stellt sich immer mehr die Frage, in welcher Weise die technologischen Möglichkeiten und die Umweltveränderungen letztendlich dazu führen, dass sich Hochschulen in ihren Arbeitsweisen weiterentwickeln und dabei darüber nachdenken müssen, in wieweit Innovation tatsächlich in der Hochschule möglich sein wird (Schünemann \& Budde 2018). Und so ist es auch bei Hochschulen insbesondere ein 
externer Innovationsdruck, der zum Gefühlten 5 vor 12 führt. Innovation in der Hochschule über Technologie, beispielsweise das freie zur Verfügung stellen von Bildungsinhalten über offene Onlinekurse, das Entwickeln von Onlinekursen für sehr große Zielgruppen (wie Massive Open Online Courses), die frei verfügbaren Bildungsmaterialien (Open Educational Resources), das alles und auch das modulare zur Verfügung stellen von Zertifizierungskonzeptionen (über sog. Badges und Microcredentials) wird durch Technologie ermöglicht und war bis vor kurzem in Hochschulen noch undenkbar. All diese Beispiele für Innovationen sind Beispiele für Innovationen in Hochschulen, die über Impulse von außen in die Hochschulen hineinkommen. Beispiele sind Coursera oder Odassity in den USA oder auch die MOOC-Plattform Future Learn, als Ausgründung der Open University UK in England. Alles Plattformen auf denen offene Bildungsmaterialien höchster Qualität weitgehend kostenlos und ohne Einschreibepflicht angeboten werden. Studierende können diese frei verfügbar in Anspruch nehmen. Alles Entwicklungen, die außerhalb von Hochschulen gegründet wurden, da nur so die Souveränität und Hoheit hochschulischen Handelns gewährleistet werden konnte. Und gleichzeitig stellen alle Entwicklungen die bisherige Funktionsweise so stark in Frage, dass ein Aufbau der jeweiligen Plattformen innerhalb der Hochschulen nicht funktioniert hat.

Insgesamt erlaubt Digitalisierung neue Vertriebs- und Informationswege, neue und nicht mehr an Institutionen gebundene, übergreifende Cloud-Datenspeicher, neue Möglichkeiten über intelligente, lernende Algorithmen den Aufbau und die Abläufe im Hochschulbereich neu zu denken. In dieser neuen, oft auch fälschlich glitzernden Welt, gilt trotzdem der berühmte Satz von John Nalsbitt: „We are drowning in information, but starved for knowledge“ - und so möge man hinzufügen „wisdom“. Indem Digitalisierung Entkoppelung und Dezentralisierung ermöglicht, ja befördert, stellen sich für Bildungsinstitutionen Fragen nach ganzheitlichen Bildungskonzepten im Duktus nicht-fragmentierter, durchgängiger und orientierungsgebender, überdauernder Weise neu und drängender.

\section{1.1.3 Digital oder analog: Was bildet besser?}

Eine Frage, die im Zusammenhang mit digitaler Lehre in der Vergangenheit oft gestellt wurde, ist die Frage, was besser sei: digitale oder analoge Hochschulbildung. Mittlerweile gibt es zu dieser Frage eine Vielzahl an Untersuchungen und eine führende Lehrmeinung. Im Kern solcher Forschungen stand immer die Frage nach der lernförderlichen Beschaffenheit von E-Learning und digitalen Medien und auch die Frage, ob mit mediengestützten Lernsystemen erfolgreicher oder effektiver gelernt werden kann als auf anderen, etwa konventionellen Wegen. Mithilfe von 
Metaanalysen können die vielen vorliegenden Untersuchungen über die Effektivität des Computereinsatzes zum Lehren und Lernen aggregiert werden. Dabei weisen Kerres und Gorhahn (1999) auf folgende Tendenzen hin:

1. E-Learning ist konventionellem Lernen nicht grundsätzlich unterlegen. Die genannten Studien konnten auch kein bestimmtes Mediensystem als besonders erfolgreich identifizieren.

2. Der Vorteil des multimedialen Lernens liegt nicht in der gleichzeitigen Ansprache mehrerer Sinneskanäle (man nennt dies naive Summierungshypothese, wie Weidemann es 1997 betitelt), sondern in der verschiedenartigen Codierung von Informationen in unterschiedlichen Symbolsystemen.

3. Die Lernmotivation lässt sich kurzzeitig durch den Einsatz von Lernmedien steigern. Da dieser Effekt aber von kurzer Dauer ist, rechtfertigt er nicht eine teure Produktion multimedialer Inhalte.

4. Insgesamt scheint viel mehr die Beschaffenheit des didaktisch methodischen Lernarrangements von Bedeutung für den Lernerfolg zu sein und weniger das eingesetzte Mediensystem.

5. Bei Personen mit hoher Lernkompetenz und selbstständigem Lernverhalten, haben Mediensysteme Vorteile gegenüber konventionellen Lernmethoden.

Eine der bedeutendsten Metaanalysen in diesem Zusammenhang stammt von Kulik und Kulik (1991) bereits aus den 90er Jahren. Die Autoren haben insgesamt 248 Vergleichsstudien ausgewertet. Davon waren bereits 195 in früheren Metastudien zusammengefasst worden und 53 kamen als damals aktuelle Studien später noch hinzu. Von den 248 Studien wiesen 202 einen höheren Lernerfolg für das computerbasierte Lernen und 46 ein besseres Ergebnis für das konventionelle Lernen aus. Die Ergebnisse waren aber nur in 100 Fällen signifikant, in 94 Prozent der Fälle zugunsten des computerbasierten Lernens und in 6 Prozent der Fälle zugunsten des konventionellen Unterrichts. Vergleichsstudien zwischen konventionellem und mediengestütztem Lernen sind nicht eindeutig in die eine oder andere Richtung auszulegen. Es gilt nach wie vor das Primat der Didaktik, welches den höchsten Einfluss auf den Lernerfolg zu haben scheint und weniger der Einfluss des digitalen Lernsystems. Thomas Russel (2001) kommt zu dem Schluss, dass im Vergleich zwischen konventionellem und digitalem Lernen, das sogenannte no significant difference phenomenon Gültigkeit hat, sodass eine Überlegenheit des einen zum anderen System nicht überdauernd festgestellt werden kann. Vergleichsstudien zwischen konventionellem und mediengestütztem Lernen sind jedoch nicht unumstritten; zum einen treffen sie die explizite Annahme, dass die zu vermittelnden Lerninhalte gleichermaßen für konventionelles Lernen und für E-Learning geeignet sind, 
zum anderen sind sie methodologisch problematisch. Fraglich ist vor allem, ob die Unterschiede wirklich in jedem Fall auf die eingesetzten Medien zurückzuführen sind. Insbesondere Variablen, die sich auf die Eigenschaften der Lernenden selber beziehen (Lernpräferenzen, Lernkompetenzen, Motivation, etc.), scheinen einen Einfluss auf den Lernerfolg zu haben. In der empirischen Lehr-Lern-Forschung wird seit längerem versucht festzustellen, welche Personen mit welchen medialen didaktischen Angeboten am besten lernen. Die Intention dabei ist, alle relevanten Einflussfaktoren einer Lehr-Lern-Situation zu erfassen und deren Wirkung auf den Lernprozess festzustellen. Unter methodologischen Gesichtspunkten bedeutet dies, dass Medienattribute wie beispielsweise Lesbarkeit der Texte, Filmsequenzen usw. sowie Variablen des didaktischen Designs in Beziehung zu den Lernvariablen gesetzt werden. Dieses Vorhaben mündet zumeist in sehr komplexen experimentellen Forschungsdesigns. Problematisch ist dabei nicht nur die Fülle der zu erfassenden Faktoren, sondern auch deren wechselseitige Einflüsse.

Insgesamt kann mittlerweile festgehalten werden, dass die Hoffnung alle bedeutenden Einflussfaktoren zu erfassen und mithilfe statistischer Methoden auf ihre Wirkung stoßen zu wollen, als unrealistisch aufgegeben wurde. In jüngster Zeit werden diese Versuche erneut belebt, indem unter dem Stichwort "Learning Analytics“ versucht wird, möglichst viele Daten des lernenden Verhaltens aufzuzeichnen und über Data-Mining-Verfahren und lernende Algorithmen, Schlüsse darauf zu ziehen, in welcher Weise Lernerfolg zu beobachten ist und wie Lernerfolge verlaufen. Auch hierbei wird über empirische Verfahren versucht, Verhaltensdaten und -variablen mit Attributen der Lernsituation wie Medien, eingesetzten Materialien und Variablen der Lernenden in Beziehung zu setzen, sodass prinzipiell kein Unterschied zu früheren Versuchen beseht, jedoch graduell in der Vielzahl der zur Verfügung stehenden Daten andere Ansätze gewählt werden können. Das Hochschulforum Digitalisierung, eine Netzwerkeinrichtung von deutschen Hochschulen, die im Bereich der Digitalisierung der Hochschullehre arbeitet, kommt zu dem Schluss, dass die Frage nicht mehr lauten kann, ob digitale oder analoge Hochschulbildung besser oder schlechter sei, sondern lauten muss, wie sie in der Zukunft gestaltet werden kann. Dabei geht es nicht um eine Digitalisierung per se, sondern es geht immer stärker um die Frage in welcher Weise digitale Medien auf den Lernprozess wirken, in welcher Weise digitale Medien den Studienprozess individueller und flexibler gestalten können und Mehrwerte aus Sicht der Lehrenden und Lernenden geboten werden können. Es stehen bei der derzeitigen Diskussion in Hochschulen drei Thesen im Vordergrund.

1. Digitalisierung ist keine Technisierung, sondern didaktische curriculare und organisatorische Innovation. 
2. Der Schlüssel zur erfolgreichen Digitalisierung der Hochschullehre ist die Kollaboration.

3. Digitalisierung schafft nicht nur virtuelle Lernräume, sondern verändert auch bestehende physische Lernräume.

\section{1.1.4 Open Education: Neue digitale Offenheit}

Digitalisierung ermöglicht eine neue, zuvor nicht dagewesene Offenheit in vielen Aspekten. Die neue digitale Offenheit, beispielsweise des offenen Publizierens, aus dem heraus neue kollaborative Arbeits- und Publikationsformen entstehen, war so in der Wissenschaft bislang nicht existent. War früher das Publizieren von wissenschaftlichen Texten eine sehr exklusive Arbeitsweise von einem oder mehreren Wissenschaftlerinnen und Wissenschaftlern untereinander in einer geschlossenen Gruppe ohne die Ergebnisse im Vorhinein nach außen darzustellen, in einer besonderen Textsorte den besonderen Qualitätsansprüchen genügen musste, so ist heute digitale Kollaboration an wissenschaftlichen Analysen ein Vorgehen in dem mit neuer Offenheit Peers bereits im Produktionsprozess des Textes einbezogen werden.

Andere Aspekte der Offenheit durch digitale Medien sind die Öffnung von Lehrangeboten für andere Zielgruppen, das zur Verfügung stellen und nutzen von Lehrmaterialien als offene Bildungsmaterialien, auch als Open Educational Resources bezeichnet (OER). Zu den offenen Bildungsressourcen zahlen alle Arten von Materialien, alle Inhalte und Konzepte, welche zu Lehr- und Lernzwecken entwickelt wurden und die ohne oder mit nur geringen Einschränkungen verwendet, bearbeitet und weitergegeben werden dürfen (vgl. Butcher 2013: 6). Sie stellen eine zeitgemäße Möglichkeit dar, die notwendigen Voraussetzungen für Bildung im Sinne des Austauschs von Gedanken, Erfahrungen und Wissen zu schaffen. Dazu wird das Material von den Rechteinhabenden in der Regel kostenlos zur Verfügung gestellt und mit einer offenen Lizenz gekennzeichnet, die eine rechtssichere, pauschale Nutzungserlaubnis umfasst. Laut UNESCO Können offene Bildungsressourcen unmittelbar wie mittelbar dazu beitragen, den Zugang zu lebensbegleitendem Lernen zu erleichtern: Durch ihre kostenlose und freie Verfügbarkeit würden gerade einkommensschwache Menschen und Bildungseinrichtungen mit begrenzten finanziellen Mitteln von OER profitieren können. Durch die Verbreitung von OER in digitalen Formaten und ihre entsprechende Verfügbarkeit könnten den Lernenden dabei raum- und zeitunabhängige Möglichkeiten der Weiterbildung gemäß dem eigenen Bedarf eröffnet werden (vgl. UNESCO 2017: 2).

Auch das zur Verfügung stellen und in Anspruch nehmen von Datenbeständen und Informationen, die als offene Daten (Open Access) verfügbar gemacht werden 
ist möglich. Insgesamt ändert die digitale Technologie auf diese Weise sowohl den Forschungsprozess (e-science), als auch die Analysemöglichkeiten über Daten, die Dokumentation, die Lehre und die Verfügbarkeit von Lehrmaterialen.

Im Bereich der digitalen Lehre der Hochschule stellt sich die Frage, was einen geeigneten, angemessenen „Blend“ von digitalen und nicht digitalen Phasen und Ansätzen für die digitale Hochschullehre darstellt. Obwohl diese Frage in allen Hochschulen, gemäß der jeweiligen Hochschulprofile kleinerer und größerer Hochschulen, der jeweiligen Fachkonstellation unterschiedlich diskutiert wird, so scheinen sich in deutschen Hochschulen derzeit doch vor allem zwei Modelle durchzusetzen: Blended Learning und die Untervariante des Blended Learning mit dem Titel Flipped oder Inverted Classroom (siehe hierzu ausführlicher Ehlers \& Kellermann 2019). Die Diskussionen in Hochschulen laufen zumeist auf Fachebene, da jedes Fach beziehungsweise jeder Lehrende eine individuelle Designentscheidung treffen kann und treffen muss, inwieweit digitale Medien und in Präsenz stattfindende Lehre miteinander zu neuen didaktischen Mustern verwoben werden. Dabei steht im Vordergrund die Funktion der Wissensvermittlung tendenziell und zunehmend mehr auf die Interaktion mit Medien zu verlagern während die Wissensvertiefung, die Anwendung des Wissens, die Weiterentwicklung und die Analyse des Wissens in spezifischen Komplexen, Fallkonstellationen und Problemsituationen im Bereich der Präsenzlehre im Vordergrund stehen.

\section{1.1.5 Der Wettlauf zwischen Bildungssystem und Technologie}

Betrachtet man die Digitalisierung als Megatrend im Zusammenhang mit der Bildung unter historischer Perspektive, so wird mit Katz und Goldin (2009) deutlich, dass ein Zusammenhang besteht zwischen gesellschaftlicher Entwicklung, Technologie und Bildungsentwicklung (siehe Abbildung 25). War es noch in der vorindustriellen Zeit so, dass zwischen technologischer Entwicklung und Bildungsentwicklung nur wenige erkennbare Zusammenhänge bestanden, wurde mit der Technologieentwicklung der Dampfmaschinen, der neuen Produktionsmittel in der industriellen Revolution, ein wirtschaftlich großer Vorsprung erzielt, dem das öffentliche Schulsystem und die zur Verfügung stehenden Bildungsressourcen und Bildungsprozesse allerdings nicht nachkommen konnten. Katz und Goldin sprechen hier von einem sich entwickelnden sozialen gesellschaftlichen Spannungsfeld, welches aus einer starken Technologieentwicklung entstand, die Produktionskapazitäten und die Produktionsressourcen sowie die Arbeitnehmerinnen und Arbeitnehmer in den Fabriken stark betraf und gleichzeitig einer nicht zur Verfügung stehenden Aus- und Weiterbildung. Erst mit der Einführung des universellen, öffentlichen Schulsystems 
bestand die Möglichkeit, auch die Bildungslevel der Gesellschaft entsprechend weiterzuentwickeln. Dabei gab es zunächst eine Phase in die Bildungsangebote massiv ausgeweitet wurden, in den fünfziger und sechziger Jahren des letzten Jahrhunderts und in der durch gut ausgebaute technologische Produktions- und Wirtschaftsmechanismen eine Phase gesellschaftlicher Prosperität entstanden. Es konnte so eine gesellschaftliche Entwicklung in Richtung verstärkter Bildung und Ausbildung zu gesellschaftlicher Entwicklung, Prosperität und neuen Lebensformen führen. Katz und Goldin sprechen hier von der Phase des Wohlstandes.

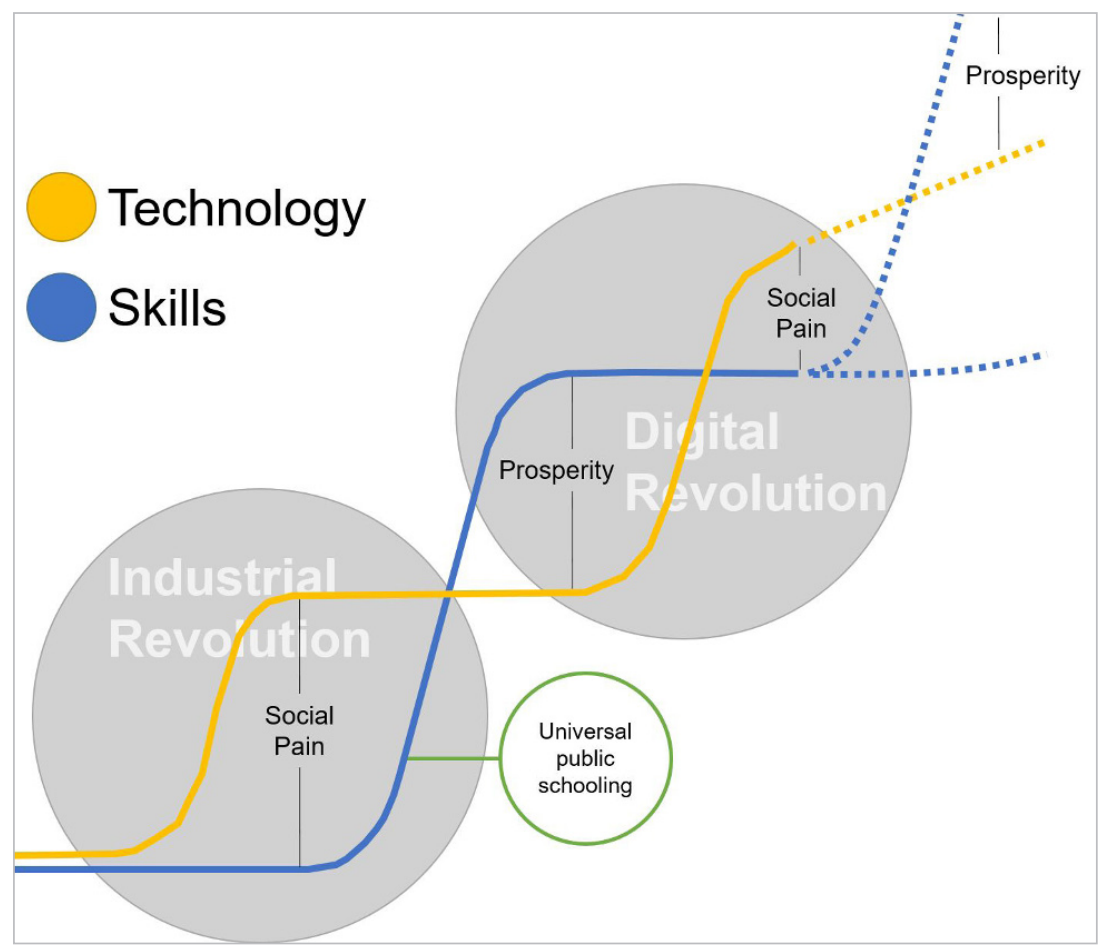

Abb. 25 Wettlauf zwischen Technologieentwicklung und Bildungssystem (Quelle: Katz \& Goldin 2009)

Mit der digitalen Revolution sehen sie erneut eine Phase, in der technologische Entwicklungen einen rapiden Entwicklungsvorsprung machen, ohne dass im Moment eine ähnliche Bildungsentwicklung eingegangen wird, ohne dass im Moment neue 
Bildungsprozesse und Bildungsformen diskutiert werden. Hierbei, genau an dieser Stelle, hakt auch das Konzept der Future Skills ein und stellt die Frage: Wie muss die neue Bildung, wie muss die neue Hochschulbildung der Zukunft eigentlich aussehen, um in gleicher Weise nach der industriellen Revolution auch die digitale Revolution zu begleiten, um nicht die Gefahr der gesellschaftlichen Irritation zu groß werden zu lassen, um sozusagen zu gesellschaftlicher Kohärenz beizutragen?

\section{1.2 Zweite Sekunde: Hochschulbildung in der (medialen) Transformationsgesellschaft}

Die Medienentwicklung hat immer schon in historischer Betrachtung zu grundlegenden gesellschaftlichen Umbrüchen geführt. Alle Gesellschaften haben immer in Umbrüchen gestanden, anhand der verfügbaren Entwicklung von Medien. Dirk Bäcker, ein Soziologe an der Universität Witten-Herdecke, zeigt dies in seiner Medienarchäologie (2018) auf, in der er vier Medienepochen unterscheidet. Die erste Medienepoche ist der Übergang in die orale Gesellschaft. Bäcker stellt die Frage: Vollenden Computer die Moderne? Versprechen sie noch immer Freiheit und Teilhabe? Oder sind wir gefangen in ihren Netzen?

Dirk Bäcker erkennt in der Digitalisierung die jüngste von vier Medienepochen der Menschheitsgeschichte, von denen jede einzelne die Regeln des Zusammenlebens grundlegend neu geprägt hat. Digitalisierung heißt die Verwendung von elektronischen Apparaten aller Art. Sie ist von derselben tiefgreifenden Bedeutung für die gesellschaftliche Kultur wie zuvor die Einführung des Buchdrucks, davor die Einführung der Schrift und davor die Einführung der Sprache. In seinem Buch „4.0 oder die Lücke die der Rechner lässt“ (2018) skizziert der Soziologe wie mit dem Auftauchen der Sprache im Zeitraum vor dreißig- bis vierzigtausend Jahren - nach seiner Zählung in der Medienepoche 1.0 - zugleich gesellschaftliche Formationen entstanden, die als Sprachgemeinschaft jeweils eigene Regeln dafür fanden, welches Sprechen in welcher Situation, unter welchen Akteurinnen und Akteuren als angemessen galt. In der Medienepoche 2.0, die mit der Erfindung der Schrift vor etwa achttausend Jahren begann, entstand durch die Möglichkeit das flüchtige Sprechen zu fixieren und zu analysieren auch eine neue Auffassung von Zeit. Bäcker dazu:

„Die Gesellschaft explodiert in Zeithorizonte. Zu schreiben heißt lesen zu können, was man gestern aufgeschrieben hat. Aufzuschreiben was man morgen lesen muss. So dass plötzlich Begriffe wie Vergangenheit, Gegenwart und Zukunft überhaupt nötig wurden." Bäcker (2018) 
Mit der Erfindung des Buchdrucks setzt Mitte des 15. Jahrhunderts die Medienepoche 3.0 ein. In ihrem Zuge wandelte sich das Verständnis von Öffentlichkeit tiefgreifend, sagt Bäcker: „die moderne Buchdruckgesellschaft ist eine in der jeder, jeden jederzeit kritisieren kann und man das sogar aushalten muss, weil die alle gelesen haben und einfach drauflos „plappern.“ (ebenda) Diese neue Vielstimmigkeit erschien nicht wenigen Zeitgenossen chaotisch und riskant. Einen Vorschlag wie sie einzudämmen wäre, macht etwa der Philosoph Immanuel Kant in seiner Schrift „Was heißt Aufklärung?“ (1784). Dort gibt er die Empfehlung, dass eine Gelehrte, beziehungsweise ein Gelehrter nur dann das Wort ergreifen sollte, wenn mindestens eine weitere Gelehrte/ ein weiterer Gelehrter anwesend sei, die/ der sie/ ihn bei Bedarf korrigieren könne. Schon bald begannen mehr oder weniger belesene Bürgerinnen und Bürger damit, die eigene Zeitungslektüre in Salons oder an Stammtischen zum Besten zu geben, zu debattieren und sich gegenseitig zu kritisieren. So sei eine viel lebendigere weitgehend ungeregelte Öffentlichkeit entstanden, sagt Dirk Bäcker, die schon auf die heutige vorausweise.

Was aber unterscheidet die derzeitige digital geprägte Öffentlichkeit der Medienepoche 4.0 ganz wesentlich von ihren Vorläufern? Die Situation in der wir heute sind, ist, dass der Stammtisch in die allgemeine Öffentlichkeit verlängert wird und man unkontrolliert jeden $\mathrm{x}$-beliebigen Kommentar, der irgendjemandem irgendwo durch den Kopf geht als Posting auf den Plattformen der Welt wiederfindet. Es ist eine andere Situation, weil es die Autoritäten, die akzeptierte Meinung, die Kanäle in denen gebündelt werden kann, was gebündelt werden muss, nicht mehr gibt. Sind wir wirklich viel anfälliger für Fälschung und Verdrehung der Wahrheit geworden als es noch die Buchdruckgesellschaft mit ihren Prinzipien der Nachprüfbarkeit und entsprechend Instanzen der Kontrolle war? Fake News sind in diesem Sinne eigentlich kein neues Phänomen. Skandalträchtige Falschmeldungen gab es schon im 19. Jahrhundert und sie haben dort für Empörung gesorgt. Es ist zwar leichter geworden, Dokumente oder Bilder zu fälschen, Fehler sind aber heute auch schneller zu korrigieren, Betrug schneller aufzuklären. Entscheidend, so Bäcker, ist bei der Entwicklung der Gesellschaft in Reaktion auf die Medienentwicklung, dass es eine Historie gibt, Vorbilder, einen Zeitraum. Weiterhin entscheidend ist, dass die Gesellschaft Digitalisierung nicht passiv über sich ergehen lässt, sondern den Freiraum nutzt, um innerhalb der Rahmenbedingungen die Algorithmen bereits allerorten setzend, bewusst so zu gestalten, wie wir in einer digitalen Gesellschaft leben wollen. Dieser Spielraum ist die Lücke, die der Rechner lässt.

„Keine einzige Software“, so Bäcker „kein einzelner Algorithmus kann uns sagen wie Wirtschaft oder Politik oder Familie zu funktionieren hat, sondern alle diese digitalen Apparate und elektronischen Medien müssen darauf warten, dass irgendjemand zu 
irgendetwas in der Gesellschaft eine Idee dazu hat, wie man damit umgehen kann und wozu man das gebrauchen kann." (Bäcker 2018)

Die Frage die sich stellt ist: Wie können Hochschulen ihre Studierenden und Absolventinnen und Absolventen auf die nächste Gesellschaft (Bäcker 2018) vorbereiten? Auf eine Gesellschaft, die sich durch die zuvor charakterisierten Entwicklungen auszeichnet. Was sind die Fähigkeiten, die Menschen in einer so veränderten Transformationsgesellschaft benötigen, um als globale Bürger Umwelt, Gesellschaft, soziale und wirtschaftliche Systeme mitzugestalten, proaktiv und nicht reaktiv zu agieren und Lösungen für Probleme der Zukunft zu entwickeln. Die Frage ist also: Wie muss das Mindset der Absolventinnen und Absolventen zukünftig aussehen? Es ist sicher nicht mehr allein das Wissen, was ausreicht. Wissen ist heutzutage in Datenbanken, Computern, in technologischen Netzwerken, in digitalen Netzwerken verfügbar. Darüber hinaus sind es Problemlösefähigkeiten, die Fähigkeiten und Kompetenzen der Innovation, der Kreativität, die entwickelt werden müssen, um die vielgestaltige Realität der sich entwickelnden Organisationen mitzugestalten. Zudem geht es um Achtsamkeit, um emotionale Intelligenz, ein Design Mindset und Systems Thinking, vernetztes Denken, Perspektivwechsel, das Einnehmen der Perspektive des Andren, um weiterzukommen. Es sind Geschichten wie diese die charakterisieren, was Studierende als Kompetenz, als Handlungsfähigkeit entwickeln müssen. Geschichten wie die in denen große Erfindungen gemacht wurden.

\section{1.3 Dritte Sekunde: Der demographische Wandel}

Universitäre Bildung war immer begehrt, aber nie so offen zugänglich wie derzeit. Abbildung 26 zeigt, dass seit den 50er Jahre ein kontinuierlicher Anstieg der Studierendenzahlen zu verzeichnen ist.

Durch den sehr erheblichen Anstieg der Studierendenzahlen in den 2000er Jahren bei zugleich abnehmender Stärke der Schulabgangskohorten fällt dem Bereich der akademischen Bildung eine herausragende Bedeutung für die Qualifizierung zukünftiger Fachkräftegenerationen zu. Der Trend zum Hochschulstudium ist ungeachtet aller Debatten um das Verhältnis von beruflicher und akademischer Bildung - eine gesellschaftliche Realität, die es zu gestalten gilt (siehe Abbildung 26). Die Hochschulen stehen dabei vor der Herausforderung, Antworten auf die entsprechenden gesellschaftlichen Erwartungen zu finden und sie mit ihren Bildungszielen in Einklang zu bringen. 


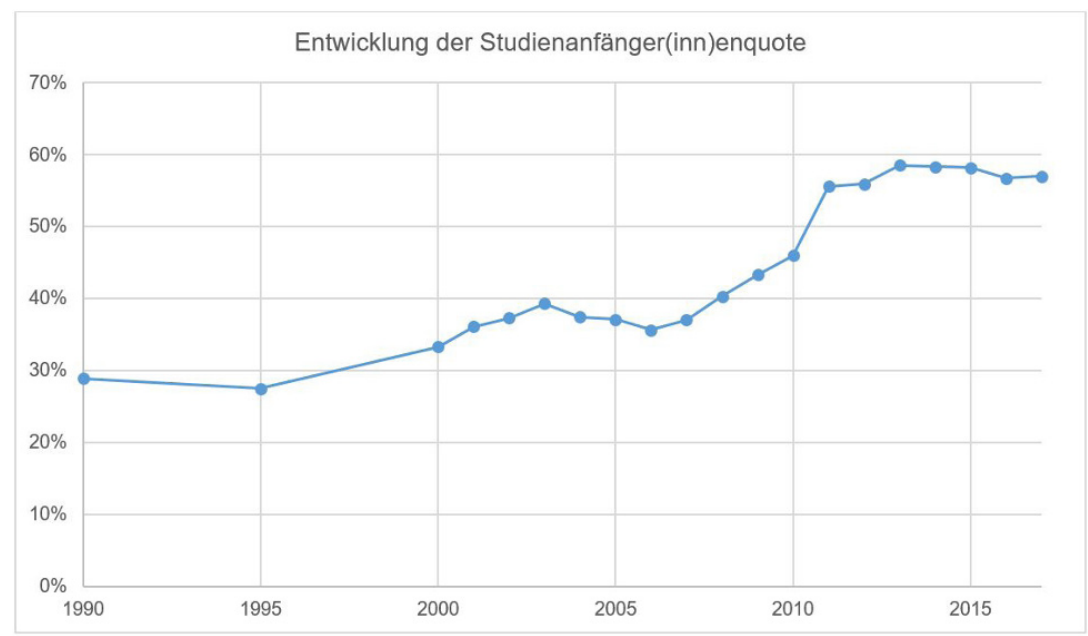

Abb. 26 Studienanfängerquote 1990 bis 2015 (Quelle: Gehrke \& Kerst 2018)

Im ersten Teil der Empfehlungen zur „Qualifizierung von Fachkräften vor dem Hintergrund des demographischen Wandels“ hat der Wissenschaftsrat nachdrücklich darauf hingewiesen, dass die Bereiche der beruflichen und der akademischen Bildung für die Qualifizierung zukünftiger Fachkräftegenerationen gleichermaßen unverzichtbar sind und in einer funktionalen Balance gehalten werden müssen. Verhindert werden soll,

„dass Jugendliche ihre Ausbildungsentscheidungen vorrangig aus Prestige-, Anerkennungs- oder Akzeptanzgründen treffen und bestimmte attraktive Bildungsoptionen allein deswegen nicht in Betracht ziehen." (Wissenschaftsrat 2014)

War die erste Hochschulausbildung im Bologna des 11. Jahrhunderts noch sehr auf die gesellschaftlichen Eliten ausgerichtet und hoch selektiv im Zugang für nur sehr privilegierte Zielgruppen, so ist durch die Bedarfe der industrialisierten Gesellschaft ausgelöst, ein wahrer Feldzug der Massenhochschulen eingetreten. Hochschulbildung zu erlangen wird heute zur Normalbiografie und Standarderfahrung (OECD 2016). Auch in Deutschland studierende mittlerweile mehr als $50 \%$ einer Alterskohorte. Die Quote der Studienberechtigten stieg 2012 bundesweit auf 53,5\% (zu Akademisierungstrends siehe auch Alesi \& Teichler 2013), die der Studienanfängerinnen und Studienanfänger auf 54,6\%, und der Studienabsolventinnen und -absolventen auf $30 \%$ (Dräger \& Ziegele et al. 2014) (siehe Abbildung 
27). Die Prognosen sind steigend, allemal geht die Bertelsmann Stiftung bis 2050 von einem Hochplateau der Studierendenanfängerzahlen aus, das weit über dem Niveau von 2005 liegt (von Stuckrad et al. 2017).

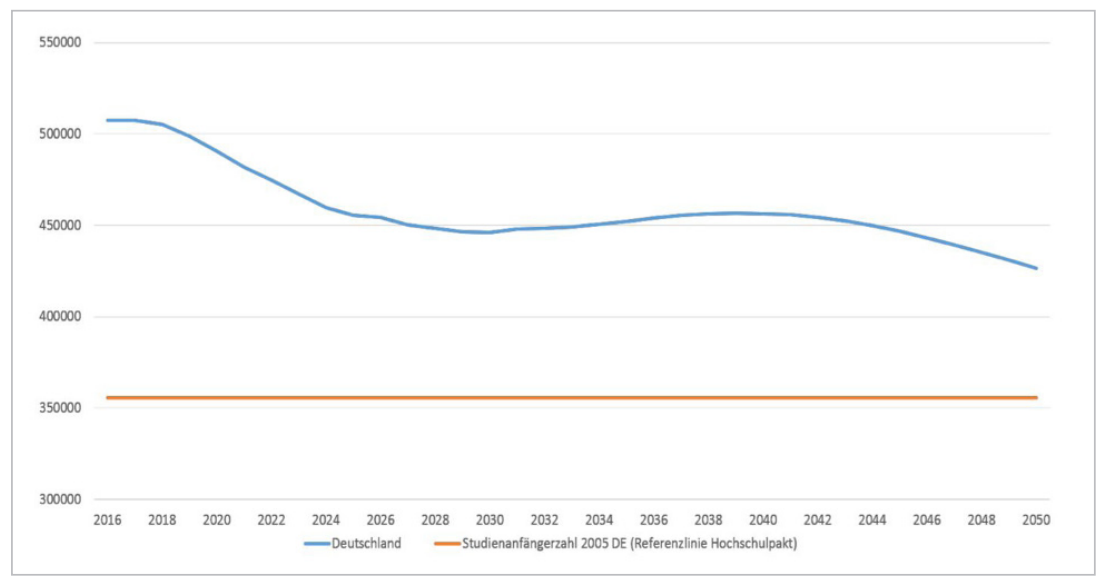

Abb. 27 Prognose Studierendenzahlen in Deutschland bis 2050 (Quelle: von Stuckrad et al. 2017)

Schofer und Meyer (2005) zeigen anhand hochschulstatistischer Auswertungen, dass die Hochschulexpansion spätestens seit der Mitte des 20. Jahrhunderts ein in allen fortgeschrittenen Ländern der Erde beschleunigt auftretender Prozess ist, der jedoch durchaus mit unterschiedlicher Geschwindigkeit verläuft. Die durchaus bedenkenswerten kritischen Interventionen zum "Akademisierungswahn“ sind demnach wichtige Reflexionsmomente, die jedoch am Faktum der stetig zunehmenden Bildungspartizipation nichts ändern (werden). Mit einer Hochschulpartizipationsrate deutlich oberhalb der 50 Prozent-Marke wird man somit überall rechnen müssen (siehe Abbildung 26, vgl. auch Teichler 2013; Baethge u. a. 2015).

Der Anteil der Erwerbstätigen mit Hochschulabschluss ist überproportional gewachsen. Betrug er im Jahr 1993 noch 13,1\%, waren es 2013 bereits 19,2\%. Der Anteil der Absolventinnen und Absolventen von Fachhochschulen ist relativ betrachtet noch etwas schneller angestiegen als der der Absolventinnen und Absolventen mit Universitätsabschluss. Der überproportionale Zuwachs an akademisch Qualifizierten betrifft alle Erwerbsformen. Unter Selbständigen sowie unter Beamtinnen und Beamten stieg der Anteil allein zwischen 2005 und 2012 um jeweils 
$12 \%$, in der Gruppe „Angestellte/Arbeiter" sogar um 16\% (Bundesinstitut für Berufsbildung 2013). Angesichts eines Akademikeranteils von rund $30 \%$ an den auf den Arbeitsmarkt nachrückenden Generationen wird sich diese Entwicklung in den kommenden Jahren voraussichtlich noch einmal deutlich beschleunigen.

Die Bedeutung von Bildungsbeteiligung als Ermöglicher am kulturellen, sozialen und ökonomischen Kapital (Bourdieu 1982) teilhaben zu können, steigt damit stetig weiter an. Der in der Pädagogik und Soziologie zunehmend stärker diskutierte Begriff der Bildungsgesellschaft (Mayer 2000) ist hierfür kennzeichnend. Damit ist sie paradoxerweise nicht nur eine wichtige Option, sondern stellt auch zunehmend ein Risiko dar, sollte eine entsprechend Bildungsbeteiligung nicht stattfinden (können) (Beck 1986). Option und Zwang liegen damit eng beieinander.

Eine weitere große Herausforderung, vor der Hochschulen heutzutage stehen, ist die Massifizierung, die Entwicklung der Studierendenzahlen und weiterer Zielgruppen die akademische Bildung nachfragen. Die OECD geht davon aus, dass in den nächsten 20 Jahren die Akademisierungsquoten für studienbefähigte Alterskohorten auf bis zu 70 Prozent ansteigen wird. Wo wir derzeit bei knapp 50 Prozent in Deutschland liegen und in anderen Ländern etwas höher, ist doch abzusehen, dass ein massiver Zuwachs an Studierendenzahlen dazu führen wird, dass Hochschulen neue Modelle entwickeln müssen. Zum einen ist es so, dass die Diversität der Zielgruppen, die jetzt schon an die Hochschule strömen und die dann an die Hochschule strömt, stärker zunehmen wird. Zum anderen werden schlicht mehr Studierende an die Hochschulen kommen als je zuvor.

Abbildung 28 zeigt die Diversität Studierender aus dem Jahr 2012, neben den traditionell Studierenden auch nicht-traditionelle Zielgruppen. Studierenden die aus den unterschiedlichsten Lebenslagen, mit den unterschiedlichen Begabungskonzepten, die in die Hochschule kommen und ganz unterschiedliche Anforderungen, Bedürfnisse und Unterstützungsbedarfe im Studienbereich haben. Hochschulen, die sich darauf einstellen können, diese unterschiedlichen Fähigkeiten und diese unterschiedlichen Ausgangspunkte und Zielkontexte der Studierenden wertzuschätzen, werden zukünftig Hochschulen mit weitgehend erfolgreichen Absolventinnen und Absolventen sein. Hochschulen, die Schwierigkeiten haben, Studienerfahrungen in dem Sinne zu personalisieren und zu flexibilisieren, unterschiedliche Studiengeschwindigkeiten, Studienverläufe, Verzweigungsrichtungen anzubieten, diesen diversifizierten Anforderungen, Interessenslagen und Bedürfnissen nicht gerecht werden, werden mit der Anforderung an Diversifizierung, der durch den Massenansturm ausgelöst wird, überfordert sein. Schaut man einmal zurück, so ist zu sehen, dass die Hochschule eine starke Entwicklung hinter sich hat. Mit der Einrichtung des ersten europäischen Hochschulcampus in Bologna 1088, wurde ein sehr exklusives Studienmodell geboren, durch welches wenige ausgewählte, 


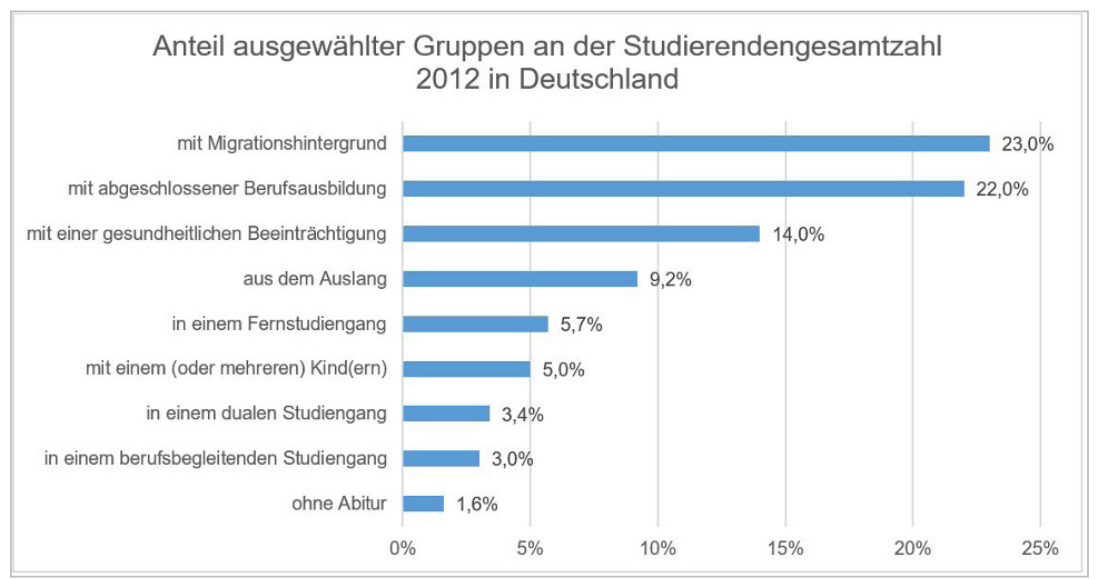

Abb. 28 Diversität Studierender (eigene Darstellung nach Dräger 2014)

privilegierte Studierende ein sehr breites akademisches Studienwissen erlangen konnten. Das Studium Generale und das Studium der Philosophie waren damals die vorherrschenden Modelle, was daher rührte, dass die Philosophie als Mutter aller Wissenschaften angesehen wurde und die dort vermittelte und geschulte logische Denkweise für alle anderen Naturwissenschaften eine Grundlage bildeten. Mit der beginnenden Industrialisierung und der dann ausgelösten gesellschaftlichen Revolution entwickelte sich auch das Bildungswesen weiter. Zunächst entwickelten sich nur die Produktionstechniken, was dazu führte, dass eine weitgehend unbefähigte Arbeiterschaft niedrige Tätigkeiten in hochgradig kleinschrittigen Produktionsprozessen unter teilweise sehr unmenschlichen und unwürdigen Bedingungen durchführen musste. Die sich so entwickelnde Wirtschaft und der damit einhergehende Wohlstand führten zu einer Bildungsexpansion, die in den 60ziger und 70ziger Jahren des letzten Jahrhunderts ihren Höhepunkt nahm und zu einer Massifizierung akademischer Bildungsangebote führte. Die Bildungsexpansion stand unter verschiedenen Zielkontexten, unter anderem auch der Förderung von bis dahin benachteiligten Zielgruppen, der Mädchen. Dies sollte dazu führen, dass immer mehr Jugendliche eine immer bessere Schulbildung und akademische Ausbildung bekämen. Dabei stand im Vordergrund, dass Qualifikationsprofile die für die Jobs in der Industrie geeignet waren, ausgebildet wurden, sodass die sich entwickelnden standardisieren Berufsprofile auch mit standardisierten Studiengängen bedient werden konnten. 
Wir können sehen, dass mit sich einer zunehmenden Massifizierung, die sich im akademischen Ausbildungsbereich abzeichnet, auch eine weitere Diversifizierung abzeichnet. Dies wird vor dem Hintergrund des sich wandelnden Paradigmas von Ausbildung auf Vorrat hin zum lebenslangen Lernen zu einer Individualisierung und zu einer notwendigen neuen Personalisierung von akademischen Studienlernverläufen führen. Die hier eintretende Individualisierung die sich im Multi Campus Studium, im Patchwork Studienverlauf mit neuen Verzweigungen und durch die Biographie ziehenden episodischen akademischen Qualifizierungsphasen ausdrücken wird, zeigt sich gelegentlich auch in Form einer postmodernen Architektur; so z. B. im Stater Center des M.I.T. oder anderen neuen Hochschulbildungsarchitekturen.

Die Durchlässigkeit beider Bildungswege - beruflich und akademisch - gilt es zu verbessern. Deshalb sollte eine abgeschlossene Berufsausbildung als Hochschulzugangsberechtigung gelten. Außerdem sollten die akademische Ausbildung mit Praxiselementen und die berufliche Ausbildung mit theoretischen Vertiefungen angereichert werden. Um auf beiden Seiten Kompetenzen und Prüfungsleistungen anrechnen zu können, müssen außerdem bundesweite Mustervereinbarungen zur gegenseitigen Anerkennung entwickelt werden.

\section{C1.4 Vierte Sekunde: Modernisierung \& Flexibilisierung des Bildungs- und Arbeitsmarkts und ein neues Verständnis von Employability}

Die Modernisierung am Arbeitsmarkt resultiert in Flexibilisierungsprozesse, Entstrukturalisierungs- und Entkoppelungsprozesse. Durch Technologisierung entstehen neue Jobs, bestehende Berufsprofile fallen weg und die Entwicklungsgeschwindigkeit der Anforderung, der Änderung innerhalb von Berufsprofilen, nimmt stetig zu. Gerade in technischen Berufen, im Bereich des Finanzwesens und in global vernetzten Tätigkeitsbereichen sind diese Zunahmen und Veränderungen sehr stark; während sie in vielen handwerklich lokal orientierten und lokal angesiedelten Tätigkeitsbereichen schwächer, aber selbst dort immer stärker spürbar werden. Dabei sind drei Entwicklungen zu beobachten:

1. Der Arbeitsmarkt entwickelt sich von einem beruflichen System der Arbeit zu einem technischen System der Arbeit (Lisop 1997). Dabei findet ein Abschied vom Berufekonstrukt als qualifikatorischer und pädagogischer Fundierung statt. Die starren Berufssysteme lösen sich mehr und mehr auf. Das technische System 
der Arbeit gewinnt an Bedeutung. Die Transformationsprozesse innerhalb eines Berufsfeldes werden intensiver, tiefgreifender und schneller.

2. Es ist eine Entwicklung vom Lifetime Employment zur Lifetime Employability zu beobachten (Beck, Giddens, Lash 1996). Dabei geht es darum, dass das Ziel von Berufsausbildung generell, aber vor allem auch von Hochschulbildung weniger eine spezifische Berufsfähigkeit eines bestimmten Berufsprofils sein kann, sondern zu einer lebenslangen Beschäftigung führen soll. Hierbei wird bereits deutlich, dass die zuvor angesprochenen Kompetenzen, die als Schlüsselstellen berufliche Handlungsfähigkeit über eigene Handlungsdispositionen bewirken sollen, eine zentrale Rolle spielen.

3. Vom verberuflichten Arbeitnehmenden zur verbetrieblichten Arbeitskraftunternehmerin/ -unternehmer (Voß, Pongratz 1998). Beck (1986) spricht hier auch von einer neuen Kultur der Selbstverständlichkeit.

In der Konsequenz ist eine unvorhersehbare rasche Entwertung starrer Qualifikationen zu beobachten, eine Entkopplung von Arbeit und Qualifikation, eine Entgrenzung von Qualifikationen und Qualifizierung. Die Globalisierung von Lerninhalten und ein verstärkter time lack zwischen dem Strukturwandel am Arbeitsmarkt und der darauf reagierenden Bildung sowie zu guter Letzt einer Ausdifferenzierung von Funktionen der Weiterbildung, um auf unterschiedliche Kontexte, die sich daran anschließen, tatsächlich auch reagieren zu können.

Neben der Flexibilisierung und Modernisierung im Arbeitsumfeld ist auch im Bildungsbereich eine Flexibilisierung zu erkennen. Hierbei handelt es sich im Wesentlichen um vier Entwicklungen:

1. Eine Flexibilisierung der Abschlüsse, die mit einer Anerkennung nicht formeller Bildung einhergeht: Die europäischen und nationalen Qualifikationsrahmen gehen davon aus, dass über eine bessere Einordnung von Bildungsabschlüssen über die gesamte Bildungskette/ Qualifikationsstufenkette hinweg eine Möglichkeit geschaffen werden soll, bruchlose Übergänge zwischen offenen Bildungssegmenten auf dieser Kette zu ermöglichen. Dabei spielt die Anerkennung von akademischer Vorqualifizierung für die akademische Weiterqualifizierung eine immer größere Rolle, die über den Bologna Prozess gewährleistet wird und auch die Anerkennung informeller und nicht formeller Bildung zukünftig werden bei gleichzeitiger Abnahme der Bedeutung von offiziellen Zertifikaten immer wichtiger.

2. Eine Flexibilisierung der Curricula und der Lernorganisation über eine Modularisierung: Immer mehr müssen Studienangebote spezifisch an die Bedürfnisse der Studierenden angepasst, angebunden sein, was nur über eine stärkere 
Modularisierung und Verzweigung sowie Wahlmöglichkeiten innerhalb der Studiencurricula erreicht werden kann.

3. Eine Flexibilisierung der Inhalte: Diese kann erreicht werden, indem weniger auf Wissen und Fakten abgestellt wird, sondern mehr auf Kompetenzen als übergreifende Handlungsdisposition in einem spezifischen, disziplinären und beruflichen Handlungskontext und auf Schlüsselqualifikation.

4. Flexibilisierung auf Ebene der Methodik der Didaktik: Die Betonung des selbstorganisierten Lernens, des selbstregulierenden Lernens und des forschenden Lernens muss zukünftig dazu führen, dass die notwendige Flexibilisierung der Studienkontexte und -abläufe, die dadurch entstehende höhere selbstverantwortete Lernleistung auch über selbstorganisiertes Lernen ermöglicht.

Wenn Wissenschaft und Wirtschaft kooperieren, profitieren in der Regel beide Seiten - allerdings gilt es, diese Kooperationen umsichtig zu gestalten. Dabei sind verschiedene Konzepte denkbar, von lose gekoppelten Partnerschaften, durch die Studierende die Möglichkeit haben, im Rahmen von Praktika, erste Praxiserfahrungen zu machen, bis hin zu strukturell hoch integrierten Modellen, wie etwa dem der Dualen Hochschule Baden-Württemberg (DHBW). Wichtig ist dabei, die Praxisstudienerfahrungen als Reflexionsgelegenheit für die Entwicklung von Kompetenzen zu verstehen, wie es etwa im Konzept zur Gestaltung von Praxisstudienphasen der DHBW angelegt ist. ${ }^{36}$

Doch wie kann man Employability noch weiterdenken - über die rein additive Praxisimprägnierung von Studierenden hinaus, in der zum theoretischen Lernen noch Praxiserfahrungen additiv hinzutreten? Wie kann ein umfassendes Konzept von Employability als Bezugskonzept für Hochschulbildungsprozesse nutzbar gemacht werden, welches weiter gefasst ist und sowohl Kompetenzentwickung, Identitätsbildung als auch Sozial- und Humankapital mit einbezieht, anstatt Qualifikationsziele aus dem aktuellen Status Quo von Berufsprofilen abzuleiten?

In der Regel sind im Studium klar definierte und unumstößlich vorgegebene Qualifikationsziele vorgegeben, die für alle Studierenden eines Studiengangs gleichermaßen zur gleichen Zeit gelten und aus denen die Inhalte und Methoden der Module im Studienverlauf abgeleitet werden. Dabei werden vielfach existierende Berufsprofile als normatives Paradigma für Studieninhalte herangezogen. Es entsteht die pragmatische Illusion, man könne die zukünftig relevanten von den

36 Mehr Informationen zur DHBW unter http://www.dhbw.de. Ein Leitfaden für die Gestaltung von Praxisstudienphasen ist ebenfalls auf den Internetseiten der DHBW verfügbar: http://www.dhbw.de/fileadmin/user_upload/Dokumente/Broschueren_Handbuch_Betriebe/DHBW_Leitlinien_Praxisphasen.pdf 
aktuell oder in der Vergangenheit wichtigen ableiten. Zu dieser Problematik tritt die vielfach verbreitete Auffassung Employability sei der Hochschulleistung und nicht der produktiven Leistung der Absolventinnen und Absolventen zuzuschreiben. Bei seiner Analyse von Employability Konzepten bemängelt Harvey (2010) die gängige Praxis der Employability Rankings von Hochschulen. Er führt an, dass Employability in Hochschulrankings nicht als den Absolventen zugeschriebene Leistung, sondern als ein Indikator für die Ausbildungsleistung der Hochschulen angesehen wird. Employability wird damit als ein Qualitätsaspekt von Hochschulen betrachtet, was zu irreführenden und widersprüchlichen Informationen führen kann (Sumanasiri et al. 2015).

Das Konzept der Employability ist mittlerweile weit entwickelt. Employability in einem umfassenden Sinne umfasst drei Dimensionen: Karriere-Identität, Anpassungsfähigkeit und Sozial- und Humankapital (Fugate et al. 2004):

- Identität (bei Fugate et al. Besonders bezogen auf „Karriere-Identität“) umfasst kognitiv-affektive Repräsentationen hinsichtlich der Erwartungen und Ziele an die eigene berufliche Entwicklung. Dieser Dimension ordnen Fugate et al. (2004) zudem auch sämtliche berufsbezogenen Persönlichkeitseigenschaften, Werte und Normen sowie Verhaltensmuster und Erfahrungen einer Person zu. - Anpassungsfähigkeit bedeutet bei Fugate et al. (2004) den Willen sowie die Selbstwirksamkeit, Wissen, Fähigkeiten und Fertigkeiten zu verändern, um den sich ändernden Anforderungen auf dem Arbeitsmarkt gerecht zu werden.

- Sozial- und Humankapital umfasst das soziale Netzwerk sowie Eigenschaften eines Individuums wie Bildung, Alter, Geschlecht, Arbeitserfahrung, Herkunft etc.

Aus der Analyse der aktuellen Karriere- und Employabilityforschung ergeben sich zwei Konsequenzen: Zum einen ist man sich in der Karriereforschung heute einige, dass Karriere und Berufsverläufe als sog. „boundaryless career“ verstanden werden müssen (Arthur und Rousseau 1996), die prinzipiell als flexibel, durchlässig und vielseitig verstanden wird. Zum zweiten steht im Mittelpunkt der Employabilityentwicklung das selbstgesteuerte und selbstorganisierte Individuum, welches für seinen beruflichen Werdegang selbst verantwortlich ist, also für die Planung und Gestaltung des Berufslebens eine wesentliche Rolle spielt (Greenhaus et al. 2011; Hirschi 2012). Hochschulen kommt dabei die Rolle einer begleitenden und anregenden Institution zu, in der Erfahrungen gemacht und reflektiert werden, die der individuellen Persönlichkeitsentwicklung im oben genannten Sinne dienen. Fachwissenschaftliche Studiengangskonzeptionen, Curricula und Lehrkonzeptionen stehen damit vor der Aufgabe, zur erweiterten Employabilityentwicklung beizutragen, indem sie sowohl Aspekte der Identitäts- und Persönlichkeitsentwicklungen 
mit einbeziehen, sich einem umfassenden Kompetenzverständnis aufschließen sowie zu guter Letzt auch die Entwicklung von Sozial- und Humankapital in den Blick nehmen.

Studieninhalte sind dabei auf Basis von Berufsprofilen an weiteren Inhalten orientiert, die eine langfristiger Beschäftigungsfähigkeit befördern: Entwicklung und Reflexion individueller Bildungsziele, Interessen und Bedürfnissen, Future Skills, grundlegende Handlungskompetenzen und die Befähigung zum Umgang mit übergreifenden Fähigkeiten.

Die Beschäftigungsfähigkeit lässt sich effektiv durch aktive und praxisbezogene Formen des Lernens fördern. Das zeigen nicht zuletzt die dualen Studiengänge, deren Abbruchquote mit nur sieben Prozent weit unter der Quote anderer Studiengänge liegt (Kupfer 2013). Dieses Erfolgsmodell sollte ausgebaut werden. Außerdem sollten Praktika in allen Studiengängen obligatorisch sein. Darüber hinaus müssen Hochschulen ein erweitertes Employabilityverständnis entwickeln, welches zumindest um eine Perspektive des global citizenship ergänzt werden sollte. Die Betonung des bewussten und verantwortungsvollen Handelns als Bürgerinnen und Bürger einer globalisierten Gesellschaft, die aktiv in die Gestaltung gesellschaftlicher Herausforderungen eingebunden sind, wie beispielsweise den Klimawandel, gesellschaftliche Potenziale durch Migration, globale politische und Finanzmarktfragen, wird für Hochschulen in Zukunft von besonderer Wichtigkeit sein.

\section{C1.5 Fünfte Sekunde: Open Education \& Shared knowledge economy}

Hochschulen sind expertenorientiere Wissens- und Bildungsorganisationen mit dem Selbstverständnis, Wissensproduktion und Wissensvermittlung strukturell miteinander zu koppeln. Durch digital offen verfügbare Wissensressourcen wird dieses Selbstverständnis hinterfragt. Insbesondere die zur Verfügungsstellung von offenen Lehrmaterialien stellt das bisherige Vorgehen von Hochschulen in Frage. Während Hochschulen sich zumeist als die alleinigen Produzentinnen, Verwalterinnen und Vermittlerinnen wissenschaftlichen Fortschritts sehen, entstehen immer mehr und andere neue Modelle, Wissen, wissenschaftliche Ergebnisse, Daten, Publikationen und Lernmaterialien verfügbar zu machen. Angelehnt an Modelle der Sharing Economy wie Uber oder Airbnb und die Möglichkeit über digitale Medien individualisierte Produkte und Prozesse skaliert an größere Zielgruppen auszubringen, wird auch im Bereich der Wissensökonomie gefragt, in welcher Weise eine Shared Knowledge Economy aussehen kann. Als 2001 der Begriff der offenen 
Bildungsmaterialien von der UNESCO auf der Bildungskonferenz in Paris entwickelt wurde, steckte die Digitalisierung aus heutiger Sicht noch in den Kinderschuhen. Mittlerweile gibt es zu fast allen Themen sowohl videobasierte als auch textbasierte Materialien, die spezifisch auf das Lernen in unterschiedlichen Bildungssegmenten (Schule, Hochschule, Weiterbildung) zugeschnitten sind. Digitalisierung erlaubt die Entkoppelung der unterschiedlichen Lehr-/ Lernservices von Hochschulen wie

1. eine Funktion des Brokerage und der Wissensproduktion: Dem Erstellen, Auswählen und zur Verfügung stellen von Lehrmaterialen und Curricula,

2. die Lehr- und Vermittlungsfunktion: Darunter fallen Lehr-, Lern- und Tutoringservices und

3. der Qualitätssicherungsfunktion, der Akkreditierung und Zertifizierung von Wissen und Kompetenzen.

Immer mehr Beispiele, gerade im privaten Hochschulbereich zeigen, dass eine Entkoppelung und Rekombinierung dieser unterschiedlichen Funktionen denkbar und möglich ist. Eine Studie von Earnest \& Young (2018) zur Zukunft der Hochschule zeigt, dass Szenarien existieren, die Hochschule anders denken und von einer Allianz der Hochschulservices ausgehen. 2011 wurde von Sebastian Thrun, einem Hamburger der als Professor in Stanford arbeitet, der erste MOOC entwickelt. Thrun, ein Wirtschaftsinformatik-Professor, entscheid sich damals, seinen Einführungskurs in die Wirtschaftsinformatik, der in Stanford 28 eingeschriebene Studierende hatte, im Internet online offen für alle Interessierten zur Verfügung zu stellen. Die sehr exklusive, selektiv ausgewählte Zielgruppe, sehr (auch finanziell) privilegierten Studierenden der Stanford University, die Thruns Kurs besuchten, schnitten insgesamt nicht so ab, wie man hätte erwarten können. Insgesamt schrieben sich 160.000 Teilnehmerinnen und Teilnehmer aus der ganzen Welt in Thruns Kurs ein. Von den 160.000 Teilnehmenden entschieden sich 23.000 am Ende an dem Abschlusstest teilzunehmen. Der Abschlusstest war durch computergestütztes Feedback hochstandardisiert. Der beste Stanford-Studierende konnte sich auf Rang 412 platzieren. Unter den ersten 20 Studierenden war auch ein kleines Mädchen aus Lahore in Pakistan, mit dem Namen Khadija Niazi, die als elfjähriges Mädchen an dem Kurs teilgenommen hatte und damit besser abschnitt als alle hochprivilegierten Stanford-Studierenden. Unter dem Gesichtspunkt der Bildungseffizienz und der Bildungsgerechtigkeit wirft dieser erste MOOC Fragen auf, die es im Zuge der digitalen shared knowledge economy zu erwägen gibt, vor allem auch unter ethischen Gesichtspunkten. Können wir es wollen, dass dauerhaft der durch digitale Medien mögliche Zugang zu Bildungsmöglichkeiten stärker geeigneten Lernenden nicht gewährt wird, solchen aber die schwächer geeignet sind schon? 
Das heißt, dass neben der Frage neuer Modelle, neuer Allianzen für eine Shared Knowledge Economy auch weitere Fragen aufgeworfen werden durch die Möglichkeit, Lehrmaterialien, Lehrservices und auch Testingservices frei zur Verfügung zu stellen. Die Frage der Bildungsgerechtigkeit zum einem indem gezeigt wird, dass durch bestehende Bildungssystemzugangsregimes nicht diejenigen studieren, die dafür am besten geeignet sind, sondern diejenigen die einen privilegierten Zugang durch Assoziation (Verwandtschaft, Beziehung) oder Ressourcen (soziales oder finanzielles Kapital nach Bourdieu) haben. Zum anderen ist damit einhergehend auch die Frage nach der Bildungseffizienz gestellt, wenn nicht mehr das Prinzip der Effizienz, sondern das Prinzip der Zugehörigkeit darüber entscheidet, welche Bildungsmöglichkeiten Individuen in Gesellschaften haben. Dies wirft zugleich die Frage nach der gesellschaftlichen Gerechtigkeit auf. Daher sind Bildungsgerechtigkeit und Bildungseffizienz aufs Intimste miteinander verwoben.

\section{1.6 Sechste Sekunde: In-Loops und Out-Loops einer lebenslangen Hochschulbildung}

Durch die angesprochene Diversifizierung und Massifizierung von Hochschulbildung wird ein seit längerer Zeit vorhandener und sich abzeichnender Trend zum notwendigen lebenslangen Lernen weiter verstärkt. Lernen wird nicht mehr im alleinigen, ausschließlichen Modell der Qualifizierung zu Beginn einer Berufsphase stattfinden, sondern Lernen wird immer mehr auch eine akademische lebensbegleitende Tätigkeit sein müssen, da sich Berufsanforderungen immer schneller entwickeln und sich auch Berufsphasen als lebenslang weiterentwickelnde wandelnde berufliche Episoden darstellen. Diese führen durchaus über zehn bis 15 unterschiedliche Stationen und münden dann erst in die Rente, die Pension oder den Ruhestand. Während lebenslanges Lernen bereits seit den 60er Jahren des vorherigen Jahrhunderts in der Bildungspolitik postuliert wird, u. a. mit dem Ziel unter Bedingungen schnellerer Innovations- und Produktzyklen gut ausgebildete Arbeitnehmende zu erhalten, wird die Forderung, Angebote für lebenslanges akademisches Lernen zu schaffen, nun auch verstärkt an Hochschulen herangetragen.

Bislang haben Hochschulen vor allem eine Konzentration auf die Qualifizierung zu Beginn einer Berufs-/ Karrierephase, zwischen High School Abschluss bzw. dem Abitur und dem Berufseinstieg. Zusätzlich sind die Fähigkeiten zur Qualifizierung, also sozusagen die Vorbereitung auf eine episodisch verlaufende Berufsbiographie in den Hochschulen vielfach nicht als Ziel akzeptiert. Vielmehr werden Curricula nach zuvor analysierten Tätigkeitsbündeln gestaltet, innerhalb von Berufsprofilen 
für die qualifiziert und ausgebildet wird. Insgesamt setzt damit das lebenslange Lernen die Hochschulen unter Zugzwang, sich sowohl inhaltlich in ihren Curricula von ihren Bildungszielen, aber auch von ihrer Bildungsstruktur von einem Modell zu Beginn hin zu einem begleitenden akademischen Bildungsmodell zu entwickeln.

\section{C1.7 Siebte Sekunde: Hochschulbildung in der VUCA Welt}

VUCA ist ein Akronym für die englischen Begriffe volatility (Volatilität, Unbeständigkeit), uncertainty (Unsicherheit), complexity (Komplexität) und ambiguity (Mehrdeutigkeit). In der NextSkills Studie geben fast neun von zehn (89,2\%) der Befragten an, dass die größte Herausforderung, auf die Hochschulen ihre Studierenden vorbereiten müssten, darin bestehe, ihnen Strategien für kontinuierliches Lernen zu vermitteln, um sich erfolgreich an sich ständig verändernde Arbeitswelten anpassen zu können $\left(M=4.17, S D=0.81, A_{\text {Adaption(strongly agree) }}=37.0 \%, A_{\text {Adaption(agree) }}=\right.$ $52.2 \%)^{37}$. Der Fokus müsse sich vom Lehren hin zum Lernen verschieben und damit einhergehend - von Lehrenden- zu Studierendenfokussierten Ansätzen, bei denen Studierende nicht als bloße Skill-Empfänger(innen) gesehen werden, sondern vielmehr zu individuellen, produktiven Lernenden, die sich eigenverantwortlich um ihre eigene Entwicklung kümmern könnten.

"Indeed, and as they [the students] are increasingly actors in their own development, they will need the capacity to steer their own learning and professional experiences."

Auch die Bedeutung im Umgang mit Unsicherheit als Bildungsziel und neues Leitbild für Hochschulbildung wurde erfragt. Die Befragten schätzten wurden den Umgang mit Unsicherheit und Ambiguität als eine der wichtigsten Skills in künftigen Arbeitskontexten ein. ${ }^{38}$ Wie aus Abbildung 29 ersichtlich, stimmten das

37 Im Delphi Fragebogen wurde dazu gebeten, folgendes Statement einzuschätzen: „The greatest challenge students need to be prepared for through higher education institutions is the continuous need for adaption through learning in changing work environments." Dazu wurden die Befragten gebeten, ihre Einschätzung auf einer 5-stufige Likert-Skala mit Werten von 1 für „strongly disagree“ bis 5 für „strongly agree“ vorzunehmen. $A_{\text {Adap- }}$ tion(strongly agree) drückt den Anteil des Samples aus, der starke Zustimmung zum Statement angibt, während $A_{\text {Adaption(agree) }}$ denjenigen Anteil angibt, der zustimmt.

38 Hierbei wurden die Befragten im Delphi Fragebogen gebeten, folgendes Statement einzuschätzen: „The ability to deal with uncertainty is the most important skill in current and future work environments." 


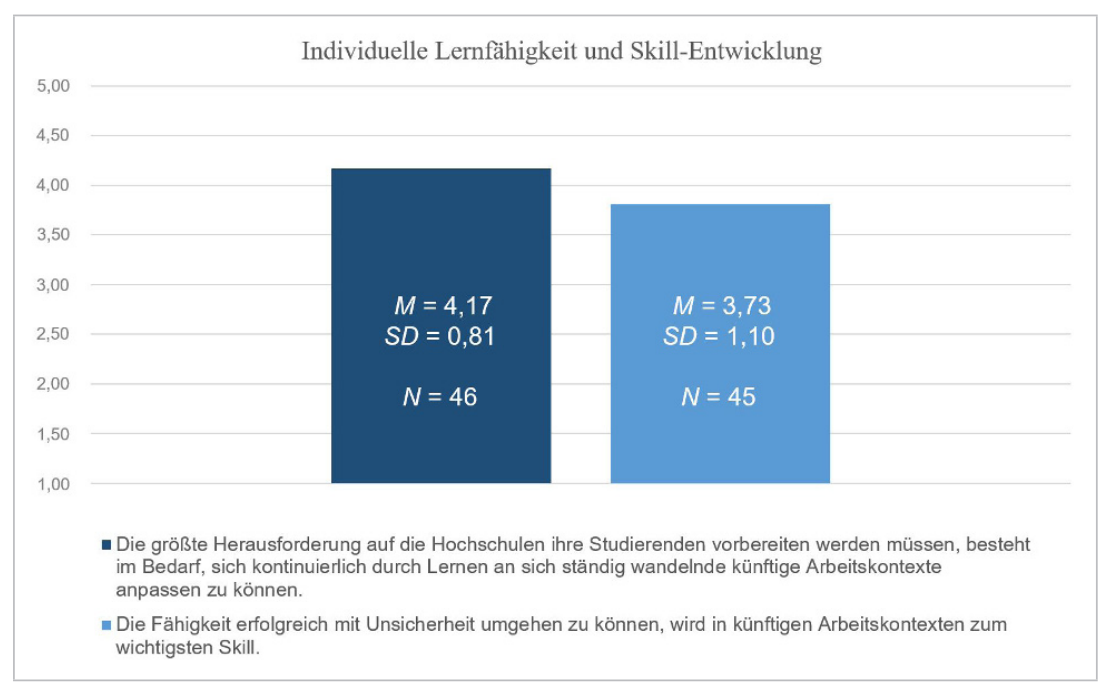

Abb. 29 Individuelle Lernerfahrung und Skill Entwicklung

Expertensample einer entsprechenden Aussage ebenfalls weitestgehend zu $(M=$ $3.73, S D=1.10, A_{\text {Uncertainty(strongly agree) }}=26.7 \%, A_{\text {Uncertainty(agree) }}=40.0 \%$ ). Die Teilnehmerinnen und Teilnehmer betonten, dass diese Fähigkeit - neben anderen Future Skills - zunehmend wichtiger werden würde und die Unterstützung von Studierenden im Umgang mit Unsicherheit in Hochschulen nicht selbstverständlich sei.

Sowohl die Fähigkeit, sich kontinuierlich durch Lernen an die sich ständig verändernde Umwelt anzupassen, als auch die Fähigkeit, erfolgreich mit Unsicherheiten umzugehen, stellen zwei zentrale Herausforderungen dar - aus Sicht der Befragten sowohl für die Hochschulen als auch für die Studierenden.

Abbildung 30 zeigt, dass für die überwiegende Mehrheit von mehr als neun von zehn Befragten die Fähigkeit, sich kontinuierlich durch Lernen anzupassen, bereits heute schon hoch relevant ist bzw. innerhalb der nächsten fünf Jahre sogar noch an Relevanz gewinnen wird (siehe Abbildung 30). Für ein gutes Drittel der Befragten wird dieser Trend wenigstens kurzfristig (innerhalb der nächsten fünf Jahre) relevant werden. 


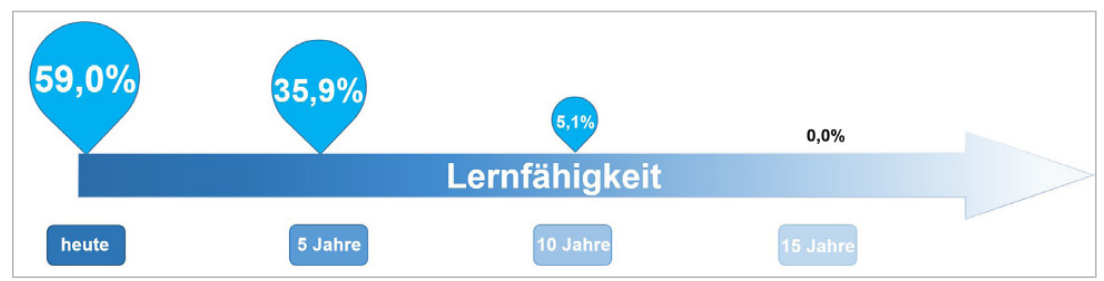

Abb. 30 Geschätzte Zeitdauer für die zunehmende Wichtigkeit der Lernfähigkeit $(\mathrm{N}=46)$

Mehr als 60 Prozent nehmen an, dass die Fähigkeit in momentanen und in künftigen Arbeitswelten erfolgreich mit Unsicherheit umzugehen, schon jetzt ein wichtiges Anliegen darstellt. Knapp ein Drittel der Befragten schätzt, dass diese Fähigkeit in den nächsten fünf Jahren an Relevanz gewinnen wird (siehe Abbildung 31).

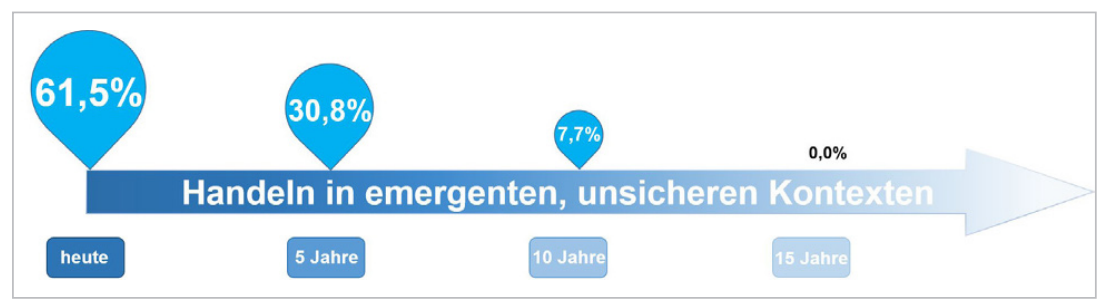

Abb. 31 Geschätzte Zeitdauer für die zunehmende Wichtigkeit der Fähigkeit in emergenten, unsicheren Kontexten handeln zu können $(\mathrm{N}=45)$

Die Modelle, die Bildungskonzeptionen und Lerntheorien, die wir benötigen, um solch gestaltende Kompetenzen herauszubilden, seit langem existieren. In der Bildungswissenschaft bezeichnet man diese Fähigkeiten als Kompetenzen. Kompetenzen sind definiert als prinzipiell unbegrenzte Dispositionen, selbstorganisiert und erfolgreich in unbekannten komplexen zukünftigen Situationen zu handeln - wie John Erpenbeck, ein Berliner Wissenschaftler, definiert. Dabei geht es nicht darum, sich dichotom von Wissen, Information und Daten abzuwenden, sondern es geht darum, Wissen, Informationen und Daten auf einer höheren Ebene zu bearbeiten und zu behandeln. Führen wir uns vor Augen, wie der Zusammenhang zwischen Wissen, Fähigkeiten, Handlungen, Kompetenzen und Professionalität aussieht (Abbildung 32). 


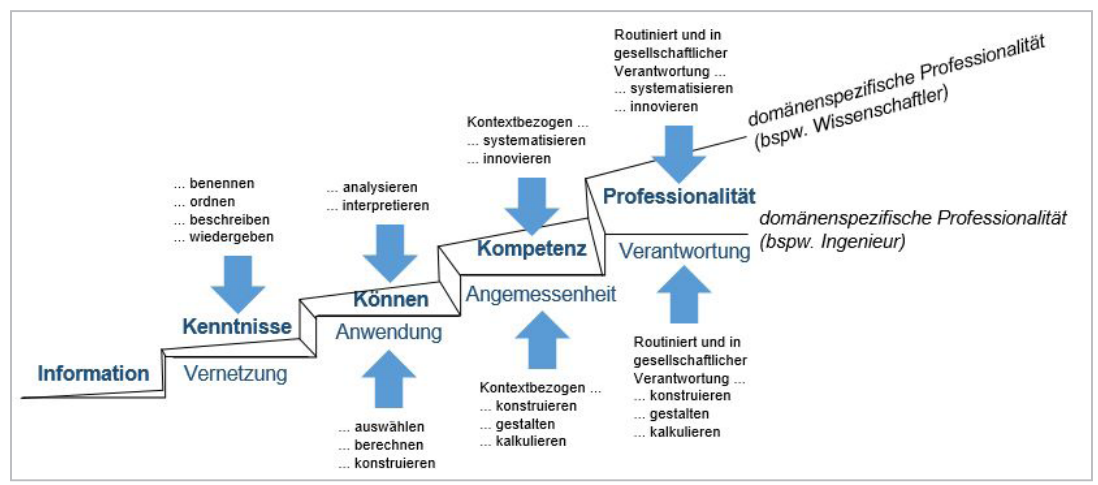

Abb. 32 Zusammenhang zwischen Wissen, Handlung und Kompetenz (eigene Abbildung nach Wildt 2006)

Die Abbildung 32 zeigt, dass erst, wenn neue Informationen mit bestehenden kognitiven Strukturen verbunden werden, wir davon reden können, dass Wissen entsteht. Erst wenn dieses Wissen angewendet wird, wir von Fähigkeiten sprechen und erst, wenn dazu noch die Volition kommt, also die Fähigkeiten etwas zu tun mit der Volition (dem Willen) und der Motivation gekoppelt ist, erst dann sprechen wir von einer Disposition zu selbstständigem Handeln. Und wenn dieses Handeln dann dem Problemkontext angemessen kontextspezifisch passiert, können wir von Kompetenzen sprechen. Werden Kompetenzen in einem letzten Schritt dann noch mit Verantwortung gepaart, so Johannes Wildt (2006), dann sprechen wir von Professionalität, der höchsten Stufe der Handlungsfähigkeit. Die Modelle diese Art von Handlungsfähigkeit zu entwickeln sind bekannt.

Ein bekanntes Modell neben vielen anderen ist das Modell des reflective practitioners. Im Modell des reflective practitioners welches Donald Schön 2006 zusammen mit Chris Argyris entwickelte, wird davon ausgegangen, dass man Reflexionsfähigkeiten erlernen kann. Schön, der in der Lehrerbildung tätig war, nimmt an, dass Lehrerinnen und Lehrer nicht auf ihre Praxissituation vorbereiten kann, weil es prinzipiell ungewiss ist, was passiert, wenn sie die Türschwelle zum Klassenraum überschreiten. Was man aber ausbilden kann, so Schön, ist die Fähigkeit ad hoc Handlungsstrategien zu entwickeln, diese zu evaluieren, zu reflektieren und herauszufinden, ob sie erfolgreich für den eigenen Fall sind. Sie möglicherweise zu überdenken und erneut auszuprobieren, zu evaluieren und nicht nur zu reagieren, sondern Handlungsstrategien weiterzudenken und wieder auszuprobieren, um sie dann ad hoc zu evaluieren und ad hoc Strategien in Handlungen umzusetzen, führt 


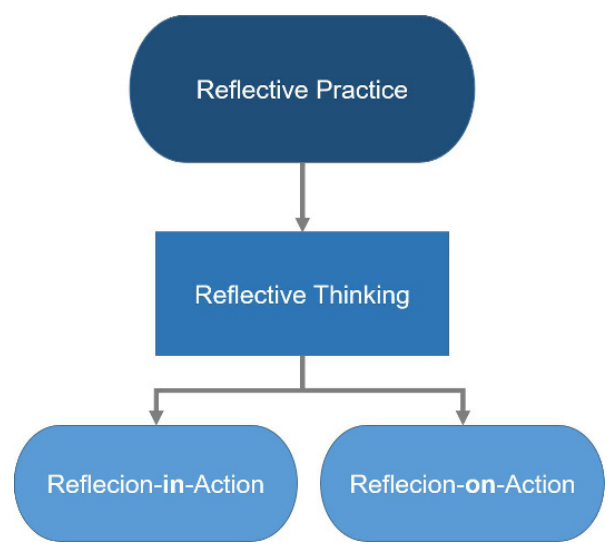

Abb. 33 Der Reflective Practitioner (eigene Darstellung nach Schön 2006)

zu einem double loop learning effect, der darin mündet, dass sich während der Handlung Reflexionsfähigkeiten entwickeln - reflection in action, so benannte Donald Schön diesen Prozess. Wenn man anfängt über diesen Prozess retrospektiv zu reflektieren, über den Prozess des reflection in action, also gewissermaßen eine reflection on reflection in action durchführt (siehe Abbildung 33), kommt man dazu, eigene individuelle Handlungstheorien zu entwickeln.

Man kommt also von der Vorannahme, einer gewissermaßen von einer automatisch vorhandenen impliziten Handlungsstrategie über eine ad hoc entwickelte Strategie in die Situation und über diese zu einer individuellen Handlungstheorie, die im Professionskontext die Professionalität, die Angemessenheit, die Verantwortung und die Volition und die Motivation entwickeln. Es ist gewissermaßen ein Zustand des perpetual beta von dem Donald Schön hier spricht, also ein Handlungszustand, in dem professionelles Handeln im spezifischen, situativen Kontext permanent mit einer Haltung stetiger Reflexion weiterentwickelt wird.

\section{C1.8 Achte Sekunde: Von der Steuerungsillusion zur Ermöglichungslogik}

Wir wissen, dass kompetenzorientiertes Lehren und Lernen vor allem in Umgebungen funktioniert, die nach soziokonstruktivistischen Prinzipien strukturiert sind. Es sind didaktische Modelle, die über das reine Faktenwissen und auch über 
die Problemlösungen hinausgehen und den Bereich von eigener kreativer selbstentwickelter und selbstverantworteter Innovation durchdringen. Insgesamt lassen sich mit Baumgartner (2004) drei unterschiedliche Lehrstrategien identifizieren (siehe Abbildung 34).

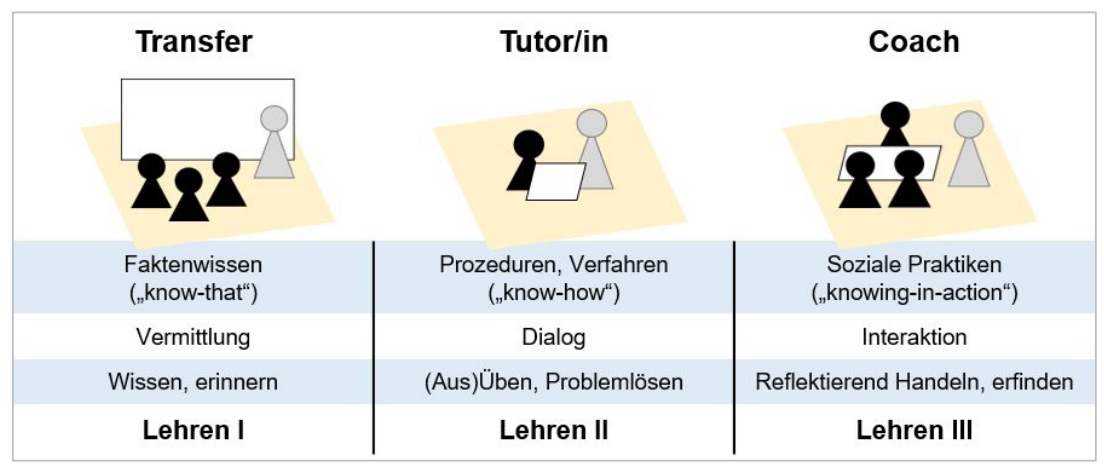

Abb. 34 Lehrstrategien (Ehlers 2010; eigene Darstellung nach Baumgartner 2004)

Modell 1 (Transfer) ist ein Bereich in dem Studierende von allwissenden Lehrenden gesagt bekommen, was sie wissen müssen. Dieses findet als Erlernen statt, als auswendig lernen und erinnern. Der Vorgang ist der Vorgang eines Vermittelns und es geht viel um Faktenwissen in der Wissensdimension des know that. In Modell 2 (Tutor/in) geht es über den Bereich des Wissens hinaus in den Bereich des Problemlösens. Das typische Modell hierfür ist das Problem-based Learning; dabei werden Studierenden Fälle von Problemen präsentiert, die sie dann als Fallbeispiel oder als Problemlöseprojekt selbstständig im Dialog mit der/ dem Lehrenden auf Augenhöhe lösen wollen. Die oder der Lehrende wird hier also zum Begleitenden, zur Expertin oder zum Experten, zur Dialogpartnerin, beziehungsweise zum Dialogpartner. Es geht vor allen Dingen darum, dass Verfahren des Problemlösens eingeübt werden, dass Prozeduren bekannt werden, es geht um prozedurales Wissen, um Know-how wodurch der Vorgang des Lehrens insbesondere als ein dialogischer Vorgang beschrieben werden kann. Das dritte Modell ist der Bereich des Coachings oder des sozialkonstruktivistischen Lernens. In diesem Modell steht das Einüben sozialer Praktiken im Vordergrund. Lehrende haben hier die Rolle nicht mehr Faktenwissen zu vermitteln oder Probleme zu präsentieren, sondern zusammen mit dem Lernenden eigene Projekte, zunächst einmal eigene Probleme zu erfinden, die dann in die Lehrsituation zurückgebracht und dort in Projekten 
gelöst werden. Es geht hier viel um echte Interaktion zwischen Partnerinnen und Partnern. Es geht darum, dass Lernende mit anderen Lernenden interagieren, dass Studierende Netzwerke gründen, sich mit Expertinnen und Experten und anderen Ressourcen verbinden. Im Vordergrund steht damit die soziale Praktik, als professionell in ein bestimmtes Professionsfeld hineinzuwachsen, so wie auch in dem Ansatz der Community of Practice von Lave und Wenger angedacht (1991). Die Logiken der unterschiedlichen Lehrmodelle folgen in den ersten Lehrmodellen eher einer Steuerungslogik, im letzteren hingegen eher einer Ermöglichungslogik. Dabei wird weggegangen von der Illusion, dass Lehren zum Lernen führt, dass es eine direkte Funktion zwischen Lehren und Lehrprozessen und Lernprozessen gibt und immer mehr eine Haltung des Respekts und der Selbstverantwortung dem Lernenden gegenüber eingenommen sowie aus der Überzeugung heraus agiert, dass lernen ein selbstverantwortender Prozess ist, in dem Lehren lediglich ein kontextueller Rahmenfaktor sein kann.

\section{1.9 Neunte Sekunde: Informelles Lernen im Studium}

Hochschulen konzentrieren sich bei der Konstruktion ihrer Lehr-Lern-Szenarien zumeist auf die formellen Lehr- und Studienaspekte. Dabei geht es beispielsweise darum, digitale Medien einzusetzen, um die Vermittlung des Wissens zu unterstützen. Der gesamte Bereich der informellen Lehre kommt dabei zu kurz. Informelles Lernen ist jedoch der Bereich, in dem nachgewiesener Weise die meisten Lernprozesse stattfinden. Es wäre eine Illusion zu glauben, dass das Studium nur aus den Lernprozessen besteht, die laut Studien- und Prüfungsordnung Relevanz besitzen. Gleichermaßen wäre es eine Illusion zu glauben, dass im Rahmen des Studiums nur in formellen Lernsettings wie beispielsweise der Lehrveranstaltung in der Hochschule gelernt wird. Ein Großteil der Lehr- und Lernprozesse findet tatsächlich als eigeninitiierte Lernaktivität der Studierenden außerhalb der formalen Lernsettings statt. Das formale Lernen findet laut der Europäischen Kommission (2001) „üblicherweise“ in einer Bildung- oder Ausbildungseinrichtung statt (in Bezug auf Lernziele, Lernzeit oder Lernförderung), ist strukturiert und führt zur Zertifizierung. Formales Lernen ist aus Sicht des Lernenden zielgerichtet. Formales Lernen ist jenes Lernen, welches im Verlauf des Hochschulstudiums schein-relevant ist und zum Zertifikat führt. Jedoch sind die vielen Anlässe informellen Lernens, die im Alltag, am Arbeitsplatz, im Familienkreis oder in der Freizeit stattfinden, oft noch mehr der intrinsischen Motivation der Studierenden entspringen. Sie bleiben oftmals jedoch abgekoppelt von dem, was in der Hochschule als offiziell prüfungsrelevant gilt. Es ist (in Bezug 
auf Lernziele, Lernzeit oder Lernförderung) nicht strukturiert und führt üblicherweise nicht zur Zertifizierung. Informelles Lernen kann zielgerichtet sein, ist jedoch in den meisten Fällen nicht intentional oder inzidentell beiläufig. Studien zeigen, dass das informelle Lernen einen großen Teil im Studium einnimmt, angefangen bei der Absprache mit Kommilitoninnen und Kommilitonen über Lern- und Studienstrategien, Lern- und Studienmaterialien, Beratung zu Lernvorhaben und Auswahl von Lernanlässen sowie das Einholen informeller Lernunterstützung im Bedarfsfalle. Die digitale Technologie spielt dabei eine zunehmend große Rolle. Bei kritischem Blick fällt auf, dass die digitalen Studienmaßnahmen der Hochschulen trotzdem häufig vor allem auf die Unterstützung der Lehre abzielen. Das heißt die Zielgruppen der Maßnahme sind vielfach in erster Linie die Lehrenden, während Studierende nur mittelbar in den Blick geraten. Obgleich der Rahmen des Hochschulstudiums zwar ein institutioneller ist, stellt das Studium einen Lernprozess dar, der sich nicht allein auf den Einfluss von Lehre zurückführen lässt. Die Perspektive der Studierenden kommt oftmals zu kurz. Die Prozesse des informellen Lernens und der Studierenden werden oft zu wenig in das gesamte Studiendesign integriert. Studien zeigen, dass Social Software Angebote wie Social Networking Sites von einer Vielzahl gerader junger Menschen genutzt werden (Busemann \& Gescheidle 2011), und dies nicht nur etwa zu privaten Zwecken, sondern durchaus auch im Rahmen ihres Studiums wie eine repräsentative Umfrage der Hochschulinformationssystem $\mathrm{GmbH}$ ergab (Kleinmann et al. 2008: 6). Demnach nutzten bereits 2008 fast die Hälfte der deutschen Studierenden Social Communities wie StudiVZ oder Facebook zum Austausch über Angelegenheiten in ihrem Studium. 2013 waren 95 Prozent der 14 bis 29 -jährigen bei Facebook angemeldet, während die VZ-Netzwerke nahezu unbedeutend geworden sind.

Auf der anderen Seite stoßen viele Einsatzszenarien von Social Software bei Studierenden auf Kompetenz- bzw. Akzeptanzprobleme (Schulmeister 2008; Jones et al. 2010). Und auch die Hochschulen und ihr Personal sind zögerlich bei der Aufnahme entsprechender Angebote im E-Learning Angebot. Eine Studie der Hochschulrektorenkonferenz HRK zu diesem Thema ergab, dass von einem flächendeckenden Transfer in die Hochschule bisher keine Rede sein könnte (HRK 2010: 35; vergleiche auch Conol 2008). Das Potential von Social Software gerade im Bereich des informellen Lernens im Studium ist dabei in der Literatur kaum strittig. Bereits zwei, kurz nach der Etablierung des Begriffes web 2.0, erschienene Beiträge von E-Learning im angelsächsischen sowie deutschsprachigen Raum bezogen die technische Innovation auf den Bildungsbereich und verwiesen auf die grundlegenden Veränderungen (Downes 2005; Kerres 2006). In der Folge wurden entsprechende Ansätze kontinuierlich weiterentwickelt (Ehlers 2013). Die größten Potentiale von Social Software liegen nach einhelliger Meinung im Bereich des 
informellen Lernens (Weigel et al. 2009). Bereits jetzt organisieren viele Lernende ihr Wissen nach Meinung der Stiftung Warentest eigenständig mithilfe von Social Software (2001).

Individualisierte Kompetenzentwicklung außerhalb formaler Lernsettings kann durch Werkzeuge wie Wikis, Blogs, e-Portfolios und Social Software wesentlich unterstützt werden (Himpsl \& Baumgartner 2009: 511). John Erpenbeck und Werner Sauter (2007) halten als Grundthese ihres Ansatzes zur Kompetenzentwicklung fest, dass es die Instrumente des Social Software sind, die ein großes Potential für die Vermittlung von Werten und Kompetenzen besitzen. Während sich traditionelle Instrumente des E-Learning dafür nur schlecht eignen (Erpenbeck \& Sauter 2007 in Ehlers 2010). Tatsächlich zeigen Studien, dass die Nutzung von Social Software durch Studierende zwar oft privat motiviert ist, der stattfindende informelle Austausch aber auch wissenschaftliche Zusammenarbeit unterstützt (Kumar, Liu \& Black 2012).

Es ist heute klar, dass informelles Lernen im Studium und in der anschließenden Berufsphase, die zukünftig immer wieder auch mit akademischen Qualifikationsphasen verbunden sein wird, einen immer weiter ansteigenden Teil informellen Lernens enthalten wird. Informellem Lernen kommt dabei als Konzept eine wesentliche Rolle zu. Informelles Lernen findet zumeist aus eigener Initiative des Lernenden statt, als selbstgesteuerter Lernprozess, aber auch viel in sozialen Zusammenhängen. Es ist klar, dass informelles Lernen einen großen Teil des gesamten Lernprozesses eines Individuums einnimmt, den größten Anteil hat. Es findet als selbstgesteuertes Lernen statt, indem Lernende ihre eigenen Lernziele festlegen und reflektieren, was sie durch Lernen an Handlungsfähigkeit erlangen wollen, ihre eigenen Lernmaterialien und Lernmethoden wählen und auch ihren eigenen Lernfortschritt überprüfen können. Informelles Lernen geht aber über selbstgesteuertes Lernen hinaus, auch in dem Bereich des inzidentellen Lernens, also des Prozesses des Anbahnens von Lernprozessen in dem Bereich der Sozialisation, des Miteinanders, des inzidentellen und des informierenden bis hin zum vertiefenden Lernen.

Als lerntheoretische Modelle spielen für informelles Lernen Ansätze, wie der des situierten Lernens, beispielsweise im Konzept der Community of Practice eine wichtige Rolle, wie es durch Lave und Wenger 1991 erarbeitet wurden. Betrachtet man das Studium unter dem Aspekt der Community of Practice, so sind Studierende Akteurinnen und Akteure im Rahmen einer Community of Practice, die sich um eine bestimmte Domäne thematisch gruppiert und eine bestimmte Praxis miteinander entwickelt, also des Lernens und Studierens in einem bestimmten Berufsfeld. Das Modell der Community of Practice wurde 2003 von Arnold auf das Fernstudium übertragen und weiter ausdifferenziert, indem er insbesondere Momente gemeinsamer Erarbeitung, gemeinsamen Lernens definiert, die sich um drei Dimensionen gruppieren (Arnold 2003): 
- Studium abschließen: Hier geht es darum, das Studium zu planen, Seminare zu besuchen, Aufgaben zu bearbeiten, Klausuren zu bestehen usw.

- Gegenseitige Studienunterstützung: Hier geht es darum, Fragen zu stellen, Antworten zu geben, Skripte zu tauschen, Lerngruppen zu organisieren, von Erfahrungen zu berichten usw.

- Kommunikations- und Kooperationsstrukturen: Hier geht es darum, digitale Medien zu nutzen, um Kommunikation aufrechtzuerhalten.

Studierende organisieren heute ihr Studium über WhatsApp-Gruppen oder gemeinsame digitale virtuelle Gruppen, teilen dort Lernmaterialien, sprechen sich in Bezug auf die Lern- und Arbeitssituation für bestimmte zu erstellende Lernleistungen ab und unterstützen sich dort gegenseitig. Studierende haben so einen sehr engen selbstgewählten, selbstorganisierten Kontakt, haben ein sehr gutes subjektives Gefühl dafür, wie in Gruppenarbeit welche Arbeiten verteilt werden, wer welche Unterstützungsbedarfe benötigt und wie viel Zeit sie mit wachsender Studienprogression, wie viel Zeit sie für welche Lernaufgaben und Lernleistung, zur Erbringung welcher Lernaufgaben, Lernleistungen benötigen. Der gesamte Bereich dieses informellen Lernens wird derzeit in Hochschulen für das Studium nur marginal erschlossen. Köhler et al. (2016) entwickeln hierfür ein Modell welches sich an dem Lebenszyklus eines Studiums orientiert und bis hin zum lebenslangen Lernen reicht, wobei sie aufzeigen, in welcher Weise Social Software Prozesse im Hochschulstudium unterstützen können. Good prächtige Beispiele:

- das Projekt „MyPaed - die persönliche Studienumgebung“ an der TU Darmstadt zum Thema "persönliche Lernumgebung“

- „KISDspaces“ der „Köln International School of Design“ zum Thema „Blogsysteme“

- „CollabUni“ der Universität Hildesheim zum Thema „Social Network“

- „E⿺辶一 $\mathrm{E}^{3}$-Protfolio Plattform Problemlösekompetenz" der Universität Augsburg zum Thema „E-Portfolios“

- „TUgether" der TU Braunschweig zum Thema „personalisierte Studierendenportale“

- „Open distributed campus“ der FU Berlin als Variante eines personalisierten Studierendenportals

Diese Beispiele zeigen in welcher Weise Hochschulen versuchen können, den Bereich des informellen Lernens durch digitale Medien zu unterstützen. Insgesamt müssen Hochschulen zukünftig zu einem breiteren Verständnis ihrer Rolle gelangen, wie sie in einer sich wandelnden Medien- und Lernwelt Lernumgebungen gestalten, 
in denen sowohl in zeitlicher als auch sozialer Dimension offene Lernräume an Bedeutung gewinnen. Dabei können digitale Medien dazu dienen, informelles Lernen im Rahmen von formellen Lernprozessen zu unterstützen. Dabei geht es darum, das Studium als Abschnitt innerhalb einer individuellen Lernbiografie zu unterstützen, während gleichzeitig spezifische soziale Lernkontexte (neben anderen auch formellen sozialen Lernkontexten) berücksichtigt werden. Nur aus dieser Perspektive können an Hochschulen Rahmenbedingungen geschaffen werden, die das selbstgesteuerte individualisierte sowie das gemeinschaftliche Lernen Studierender ausreichend unterstützen. Die durch die Digitalisierung entstehenden virtuellen Räume bieten auch aufgrund ihrer Offenheit hierfür ausreichend Potential.

Zukünftig wird es darüber hinaus wichtig, dass auch neue Formen des Studierens, neue Studienpfade durch digitale Medien unterstützt werden. Die hier veröffentlichten Ergebnisse der Delphi-Studie (Ehlers \& Kellermann 2019) (Verweis auch auf das entsprechende Kapitel, das die Delphi-Studie vorstellt) zeigen das sehr deutlich. Studium wird zukünftig als ein multiepisodischer Prozess des lebenslangen Lernens stattfinden. Studium wird darüber hinaus als zunehmend zwischen verschiedenen Hochschul-Campus stattfindender Prozess organisiert werden, indem Lehrveranstaltungen als Curriculum nicht nur an einer Hochschule erbracht und wahrgenommen werden, sondern in den Lehrveranstaltungen verschiedener Hochschulen in ein Studium hinein integriert werden und Studium sich stark flexibilisieren, individualisieren und personalisieren wird, unter anderem auch durch die Nutzung digitaler Medien, die individualisiertes selbstgesteuertes Lernen unterstützen. Dabei wird es verstärkt dazu kommen, dass Lernkontexte sich entformalisieren und durch informalisierte Anteile angereichert werden. Hierdurch kommt es zu einem immer stärkeren Blend zwischen informellen und formellen Lernkontexten. Hochschulen sind dabei aufgefordert, die informellen Lernleistungen und Lernergebnisse, die insbesondere auch lebensweltlich imprägnierte Kontexte der Studierenden mit einbringen, also situiertes Lernen in erhöhtem Maße erlauben, in das formelle Studium zu integrieren. Dies wird umso bedeutsamer werden, als dass aus Studien bekannt ist, dass auch in der späteren Berufsphase dem informellen gegenüber dem formellen Lernen eine weitaus größere Bedeutung zukommt. Das von Jay Cross (2003) aufgegriffene sogenannte „spending outcome paradox“, welches empirisch nie nachgewiesen wurde, aber konzeptuell weitgehend akzeptiert ist, zeigt auf, dass zwar 80 Prozent der Kosten für formelles Lernen anfallen, jedoch nur 20 Prozent des Lernens in entsprechenden Kontexten stattfindet. Dagegen findet 80 Prozent des Lernens in informellen Kontexten statt, für die nur 20 Prozent der Kosten aufgewendet werden. 


\section{1.10 Zehnte Sekunde: Badges \& Microcredentials}

Seit einiger Zeit sind Microcredentials, Badges, Nanodegrees und MicroMasters in aller Munde. Worum geht es dabei? Es geht dabei darum, größere Studienabschnitte in kleinere Studieneinheiten zu modularisieren und zu dokumentieren, in welcher Weise Studierende Lernerfahrungen, Wissen oder Prüfungen und Tests zu den jeweiligen Abschnitten und Modulen bestanden haben. Aus diesen kann dann eine Bildungsbiografie in viel granularerer Form und viel näher am tatsächlich Erworbenen erstellt werden. Eine wichtige Zutat dabei ist die Frage, in welcher Weise Bildungseinrichtungen, Hochschulen in der Lage sind, zukünftig Wissen nicht nur zur Verfügung zu stellen und in Form von reproduktiven Testverfahren wieder abzurufen, sondern auch Prüfungsformate zu gestalten, die Erfahrungen, Kompetenzen sowie Vorwissen aus dem akademischen und aus dem nicht akademischen Bereich abfragen und so ins Studium mit eingebracht werden können. Die zugrundeliegende Idee und Konzeption akademischer Ausbildung, die durch Microcredentials und Micro- Qualifications ermöglicht wird, besteht darin, eine lebenslange informelle Ausbildungsdokumentation, eine lebenslange Dokumentation der akademischen Ausbildung zu ermöglichen, bei der informelle und formelle Elemente, Module und Lernerfahrungen durch akkreditierte oder nicht akkreditierte, zertifizierte oder nicht zertifizierte Module miteinander zur akademischen Bildungsbiografie verwoben werden. Der CEO von Ernst \& Young, von Google, von Siemens hat bereits 2013 verkündet, dass Hochschulzertifikate wie der Bachelor oder der Master bei der Personalauswahl keine prognostische Kraft mehr in den eigenen Organisationen entfalten, sondern viel mehr auf die Persönlichkeit, auf die Erfahrungen, auf die Projekte Wert gelegt wird, die die Kandidatinnen und Kandidaten, die Bewerberinnen und Bewerber mitbringen und gemacht haben. Dabei geht es darum, wirklich gemachte Erfahrungen und Kompetenzen zu dokumentieren und miteinzubringen sowie Kompetenzen anhand von tatsächlichen Erfahrungen und Tätigkeiten Evidenz-basiert zu demonstrieren. Vielen Personalchefs in privaten und öffentlichen Organisationen werden diese Bereiche der erfahrungs- und Evidenz-basierten Kompetenzdarlegung wichtiger als die offiziellen Hochschulzertifikate. Auch die Studie von Ehlers (2018) zeigte, dass Organisationen der Meinung sind, dass Hochschulzertifikate zunehmend mehr einen Hygienefaktor im beruflichen Professionalisierungsprozess darstellen, indem sie als legale Schwelle für den Eintritt in eine Berufssphäre gelten, jedoch keine valide Aussage mehr treffen, wie gut die tatsächliche Handlungskompetenz und Performance der jeweiligen Kandidatinnen und Kandidaten im Berufsfeld eigentlich ist. Ein dazugehöriger Organisationswandel im organisationalen Gefüge, die sich sehr stark werteorientiert ausrichten und zunehmend auf Kooperation, Vernetzung 
und flache Hierarchie in einem agilen Umfeld abzielen, geht damit einher und führt dazu, das Personalauswahlprozesse immer mehr auf klein granulare beschreibbare Evidenz-basierte Erfahrungsportfolios setzen werden. Microcredentials als Leistungsnachweise entwickeln sich derzeit in verschiedenen Ländern in Europa und auf globalem Level. In den Hochschulen, um nicht nur große Studienabschnitte von 180 ECTS für einen Bachelor oder 300 ECTS für einen Master zu zertifizieren, sondern auch zunehmend mehr Kompetenzen unterhalb diese Formalebene zu zertifizieren - etwa die erworbene Kompetenz in Summer School Modellen oder kleinere Module über mehrere Wochen. Zertifikate für short courses gewinnen stark an Bedeutung. Studierende stehen vor der Herausforderung diese Microcredentials in einem evidenz-basierten, validierten Format vorzuhalten und dann eventuell in einem Bewerbungsprozess bei einer möglichen Arbeitsgeberin, einem möglichen Arbeitgeber zu präsentieren. Plattformen für solche alternative Zertifizierungsformen entwickeln sind in zunehmendem Maße verfügbar und sind dabei, Portfolios in diesem Sinne zu entwickeln. Microcredentials, informelles Lernen, Digitalisierung, Kompetenzorientierung und Flexibilisierung im Bildungsbereich sowie die Entstandardisierung am Arbeitsmarkt führen dazu, dass Hochschulbildung vor enormen Fragen steht. Ehlers (2018) schreibt dazu:

"Although alternative credentialing is just emerging, tools, platforms and concepts are already starting to emerge and develop. In technology, GitHub has become the standard platform for showcasing code to potential employers. In finance, students are using EquitySim to demonstrate trading and portfolio management skills to investment banks. Across a wide range of dynamic sectors of the economy, students are uploading papers, presentations and problem sets to Portfolium to demonstrate capabilities. And skill passports on Viridis, or digital credentials from Credly are allowing employers to find exactly the competencies they're seeking." (Ehlers 2018)

\section{C1.11 Zusammenfassung und Fazit}

Die Zukunft der Hochschule spannt sich wie ein Horizont - mit diesem Zitat von Niklas Luhmann (1976) haben wir dieses Kapitel begonnen. Luhmann (1976) beschreibt, dass in allen sozialen Systeme Erwartungen gebildet werden, die maßgeblich sind dafür, wie sich das System, auch die Hochschule, in seinen Operationen auf die Zukunft ausrichtet. Die in diesem Kapitel analysierten und beschriebenen Entwicklungen beeinflussen diese Erwartungen. Sie prägen die Situation innerhalb der Hochschule und die Erwartungen ihrer Akteurinnen und Akteure. 
Betrachtet man die Hochschulen in Deutschland so ist scheinbar alles in Ordnung. Auf den ersten Blick scheint alles gut zu laufen: Obwohl die Zahl der Studierenden in Deutschland innerhalb von nur anderthalb Jahrzehnten um eine Million auf zuletzt 2,8 Millionen gestiegen ist (Gehrke \& Kerst 2018), sind die Hochschulen nicht kollabiert. Und auch der Bologna-Prozess, der unter anderem die international anerkannten Studienabschlüsse Bachelor und Master mit sich brachte, ist so gut wie abgeschlossen. Aber es hakt im System: Die deutschen Hochschulen hinken in puncto Digitalisierung und Internationalisierung hinterher. Und auch die Lehre lässt vielerorts zu wünschen übrig, wie die teils hohen Abbruchquoten zeigen. In manchen Fächern bricht jeder zweite Studierende sein Studium ab, oft fehlt der Praxisbezug, die Auslandsmobilität stagniert.

Der Megatrend der gesellschaftlichen Entwicklung hin zu einer Bildungsgesellschaft mit all ihren Erscheinungsformen wird durch einen zweiten gesamtgesellschaftlichen Megatrend verstärkt, den der Digitalisierung (siehe Abbildung 35). In beiden Entwicklungen sind eine Reihe von Ursache-Wirkungsbündeln enthalten, die in ihren Auswirkungen starken Einfluss auf die Entwicklung der Hochschule der Zukunft nehmen.

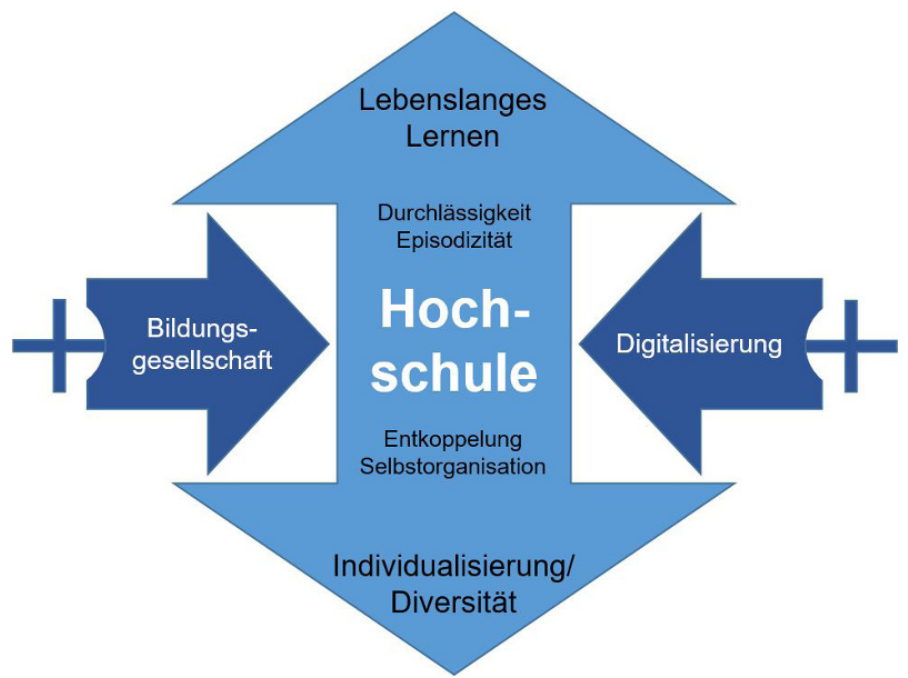

Abb. 35 Einflussfaktoren auf die Hochschule 
Beides, sowohl die gestiegene Beteiligung an akademischer Bildung als auch die zunehmende Digitalisierung der Hochschulbildung wirken wechselseitig verstärkend auf die Organisation und Ausgestaltung von Studium, Lehre und Forschung. Eine neue Vielfalt und Entkoppelungsprozesse sind die Folge und lösen einen immer stärker spürbaren Gestaltungsdruck in Richtung Individualisierung und lebenslanger akademischer Bildungsnotwendigkeit aus.

Diversität ist das große Stichwort der Hochschulbildung in jüngster Zeit. Sie wächst auf dem Zusammenhang, dass akademische Bildung einen immer größer werdenden Stellenwert für die soziale Teilhabe an der Gesellschaft besitzt, dass Bildungsprozesse zunehmend individueller (also auf den jeweiligen Bedarf der/ der einzelnen Person und Biografie zugeschnitten), und dadurch auch diversifizierter und an die jeweiligen Lebenslagen in Form und Inhalt angepasster werden (also weniger an Standartangeboten orientiert sind). Diese neue Vielfalt ist eine Heterogenität, die die große Herausforderung der Universitäten in den nächsten Jahren darstellt. Die ,klassische Klientel wissenschaftsaffiner und akademisch orientierter Studierender wird zu einer Minderheit an den Hochschulen werden. Der Bologna-Prozess gibt eine immer stärker berufsorientierte Hochschulausbildung vor, die für immer mehr Studierende der Beweggrund für ein Studium ist. Hochschulen werden sich auf die Vielfalt einstellen müssen, weil sie andernfalls weder den sich verändernden gesellschaftlichen Anforderungen gerecht werden noch ihre Studierenden verstehen können. Derzeit besteht an Hochschulen oftmals der Eindruck, dass es kein großes Problem gäbe: Die Abbruchquoten in Deutschland mit um die 25 Prozent im OECD-Durchschnitt insgesamt eher niedrig. Jedoch geht es nicht nur darum, möglichst alle Studierenden wie bisher durch die erprobten Studienkonzeptionen zu schleusen, sondern die Frage zu stellen, welche neuen Fähigkeiten und Kompetenzen die Studierenden mit ins Studium bringen und wie deren Interessen zu einer Bereicherung der Lehre beitragen könnten.

Im Umgang mit mehr Vielfalt wird es für Hochschulen wichtig, Selbststeuerungsprozesse durch Studierende zu ermöglichen, um die potenziell sehr unterschiedliche Zielstellungen einer Studienkohorte miteinander in Einklang zu bringen. Während es in einem Fall noch darum geht, ein grundständiges Studium zu absolvieren, ist es in anderen Fällen ein berufsbegleitendes oder ein praxisintegriertes Studienmodell, hier besteht vielleicht das Interesse an einem Kontaktstudium und dort an einer vertieften fundierten Studieneinheit in einem Spezialfach. Diese unterschiedlichen Bedarfe und Interessen müssen zukünftig durch intelligente und modularisierte Studienmodelle miteinander kombinierbar werden. Studierende nehmen für sich ein stärkeres Wahlverhalten in Anspruch und nutzen die Möglichkeit zum Studium aus vielfältigsten Lebenslage und Positionen im Lebenslauf. So resultiert der Studienabbruch in der Mehrheit der ersten Semester mittlerweile nicht mehr 
aus Leistungsgründen, sondern aus der Tatsache, dass Studierende sich innerhalb der ersten Studienphase umentscheiden, vielleicht ein anderes Fach studieren möchten, eine andere akademische Institution wählen oder ganz aus dem Studium aussteigen möchten, was sie vielleicht später wiederaufnehmen wollen. Um solchen Bildungsverläufen gerecht zu werden muss die Konzeption eines akademischen Studiums neu gedacht werden: Kleinere akademische Qualifikationseinheiten zu konzipieren, diese in intelligenten Weisen miteinander koppeln zu können und dabei gleichzeitig nicht die großen Qualifikationslinien aus den Augen zu verlieren. Zertifizierung, Prüfung, Examen nur noch für ein gesamtes Studium abzunehmen, das Studium aus, einer Hand, einer Institution, an einer Hochschule von A bis Z, wird zukünftig der Vergangenheit angehören oder zumindest neben das heute bekannte Normalmodell treten.

Eine dritte Entwicklung sind die sich abzeichnenden Entkoppelungsprozesse. Zum einen ist zu erkennen, dass sich die Vorstellung, die für einen Beruf notwendigen Qualifikationen und Kompetenzen ließen sich in klare und überdauernd gültige Curricula verpacken, als zunehmen absurd erweist. Es ist vielmehr eine Entwicklung von einem beruflichen und an Berufsdefinitionen orientierten System der Arbeit zum einem flexiblen System der Arbeit zu erkennen, in den Berufsdefinitionen nicht mehr starre Tätigkeitsbündel umfassen, sondern sich stetig weiterentwickeln. Lisop und Beck sprechen hierbei von einem Abschied vom „Berufe-Konstrukt als qualifikatorischer und pädagogischer Fundierung" (Lisop 1997; Beck 1986). Die Hochschule der Zukunft kann akademische Qualifikationen zukünftig nicht mehr als starres ,Paket eng umgrenzter beruflicher Qualifikationen konzipieren. Vielmehr bedingt eine industriell hoch entwickelte Struktur von Produktion, Forschung, Entwicklung und Dienstleistungen einen raschen Wechsel der Qualifikationen. In der Konsequenz sind Universitäten aufgefordert, sich mehr an übergreifenden Kompetenzen und weniger an passgenauen Qualifikationen zu orientieren.

Im Bereich der Studienorganisation zeichnen sich ebenfalls Entkoppelungsprozesse ab: So bei der Entkoppelung von Studium und Abschluss. Akademisches Studium wird zukünftig nicht ausschließlich mit dem Ziel eines Abschlusses durchgeführt werden. Vielmehr wird der Bedarf an akademischer Weiterbildung steigen, an phasenweise verfügbarer akademischer Vertiefung von beruflich relevanten Themen. Auch werden die Motive akademische Bildung als ,Genuss im Lebensvollzug in Anspruch zu nehmen wichtiger werden. In einem zunehmend digitalisierten Markt für akademische Bildungsangebote werden akademische Qualifikationen zukünftig auch nicht mehr nur noch aus einer Hand, von einer Institution und vollumfänglich betreut werden (können). Vielmehr werden Studierende auf Grundlage ihrer eigenen Präferenzen zunehmend ihre eigene Zusammenstellung von Angeboten und Institutionen vornehmen. Damit entkoppelt sich 
das akademische Studium auch von einer ,Ein-Campusmentalität, hin zu einer potenziell entkoppelten ,Viel-Campusmentalität, in der Studium und Institution voneinander getrennt zu sehen sind.

Ein weiterer Entkoppelungsvorgang ist die Entkopplung der Zeitspanne, in der ein Studium stattfindet: Akademische Qualifizierung wird zukünftig nicht mehr als,Qualifikation auf Vorrat direkt nach einem Abschluss einer weiterführenden Schule in Anspruch genommen werden, sondern in episodischen Verläufen, prinzipiell unbegrenzt über die gesamte Lebensspanne hinweg. Der Markt akademischer Weiterbildung, in dem dieses Bildungssegment derzeit angesiedelt ist, wird sich von einem Nischenmarkt (heute) zu einem Standardangebot zukünftiger Universitäten entwickeln.

Frei nach Karl Valentin sind Prognosen ja bekanntlich schwierig - vor allem, wenn sie die Zukunft betreffen. Das gilt auch für die Zukunft der Hochschule. Trotzdem ist es ein Thema, was immer wieder zu Konferenzen und Workshops inspiriert, meistens nicht so sehr, um nachzudenken, was sich ändern wird, sondern vor allem, was sich ändern sollte.

Eines wird dabei durchweg deutlich, so weitegehend und kontrovers die Diskussionen auch sein mögen: Die Universität ist nicht am Ende. Wir müssen keinen Abschied feiern. Sie ist in der Kritik und Begriffe wie ,Bildung neu denken und Digitalisierung spielen dabei wechselnde Rollen zwischen Begleiter und Treiber. Unüberhörbar ist beispielsweise auch die Kritik am Bologna Prozess: So sei bereits eine starke Verschulung der neuen Studiengänge zu erkennen. Einige Kritiker sehen in den Reformen das endgültige Ende der humboldtschen Universität, der damit verbundenen Idee von Bildung und damit das „Ende einer Lebensform“" (Seibt 2007). Auch die zunehmende, Separierung von Forschung und Lehre und die Ersetzung von ,Innensteuerung (Interesse an Inhalten) durch ,Außensteuerung (scheinorientiertes - das heißt an Leistungsnachweisen - Studium unter Zeitdruck) wird angeführt. Das bildungspolitische Ringen um den richtigen Weg zur Reform von Bildung, in Schule und Hochschule zeigt sich auch in bildungspolitischen Paradoxien: Aus der Erkenntnis, dass Bildung immer wichtiger wird, wird der Schluss gezogen, dass ein verkürztes zwölfjähriges Abitur in Zukunft ausreichen müsse und auch die Studienzeiten verkürzt werden müssten. Den Wunsch nach mehr Bildungsgerechtigkeit und mehr Hochschulabsolventen kombinierte man mit der Einführung von Studiengebühren. Größere wissenschaftliche Kompetenz versprach man sich von der Ausrichtung der Universitäten auf Drittmittelorientierung. Die Hochschule scheint derzeit allein, umringt von Reformern.

Doch lehrt uns die Geschichte, dass Entwicklung nicht zurück zu dem alten Zustand führen (sollte), sondern, dass ein neuer Zustand, der in einer Verbindung der Tradition und des Eingeübten, mit der neuen Möglichkeit liegt, anzustreben 
ist. Wie kann das für die heutige und die zukünftige Hochschule aussehen? Meine These ist, dass eine Reihe von Keytrends und Entwicklungen identifiziert werden können - unter ihnen auch eine stark erhöhte Bildungsbeteiligung und die Digitalisierung - die zu tiefgreifenden Änderungen der Konzeption der modernen Hochschule führen werden.

Als die wichtigste gesellschaftliche Institution Europas wird sich dich Hochschule behaupten können (Rüegg 1993). Sie wird sich in Organisationsstruktur und Arbeitsweise ändern müssen, will sie den geänderten Rahmenbedingungen einer Gesellschaft Rechnung tragen, in der akademischen Bildung die normalbiografische Erfahrung der Mehrheit einer Alterskohorte ist. 